\title{
Sensitivity Analysis of the Forward Electroencephalographic Problem Depending on Head Shape Variations
}

\author{
Michael Doschoris, ${ }^{1,2}$ George Dassios, ${ }^{1,2}$ and George Fragoyiannis ${ }^{1}$ \\ ${ }^{1}$ Division of Applied Mathematics, Department of Chemical Engineering, University of Patras, 26504 Patras, Greece \\ ${ }^{2}$ Institute of Chemical Engineering Sciences, Stadiou Street, P.O. Box 1414, 26504 Platani, Patras, Greece \\ Correspondence should be addressed to Michael Doschoris; mdoscho@chemeng.upatras.gr
}

Received 2 October 2014; Revised 22 December 2014; Accepted 31 December 2014

Academic Editor: Kalyana C. Veluvolu

Copyright (c) 2015 Michael Doschoris et al. This is an open access article distributed under the Creative Commons Attribution License, which permits unrestricted use, distribution, and reproduction in any medium, provided the original work is properly cited.

A crucial aspect in clinical practice is the knowledge of whether Electroencephalographic (EEG) measurements can be assigned to the functioning of the brain or to geometrical deviations of the human cranium. The present work is focused on continuing to advance understanding on how sensitive the solution of the forward EEG problem is in regard to the geometry of the head. This has been achieved by developing a novel analytic algorithm by performing a perturbation analysis in the linear regime using a homogenous spherical model. Notably, the suggested procedure provides a criterion which recognizes whether surface deformations will have an impact on EEG recordings. The presented deformations represent two major cases: (1) acquired alterations of the surface inflicted by external forces and (2) deformations of the upper part of the human head where EEG signals are recorded. Our results illustrate that neglecting geometric variations present on the heads surface leads to errors in the recorded EEG measurements less than $2 \%$. However, for severe instances of deformations combined with cortical brain activity in the vicinity of the distortion site, the errors rise to almost $25 \%$. Therefore, the accurate description of the head shape plays an important role in understanding the forward EEG problem only in these cases.

\section{Introduction}

Reconstruction of cerebral activity via Electroencephalographic (EEG) recordings is an established tool and is of great medical significance. However, its reliability strongly depends on accurate algorithms which can efficiently handle the inverse solution, which, on the other hand, essentially depends on the preciseness of the forward problem. The geometry of the brain-head model holds a decisive role allowing the installation of analytic algorithms in a very limited number of cases [1-4] where else for realistic models computer simulations have to be introduced. In any event, an important question arises: how strong does the presence of cranial deformations influence the forward problem and therefore the accurate reconstruction of the source? A precise answer is of significant importance in clinical applications where, as an example, the precise mapping of neuronal activity is a prerequisite for neurosurgical preoperative planning $[5,6]$.
As of today, computer simulations studies [7-13] have provided strong evidence on the dependence of geometrical properties of the head model used on source localization. They all agree that neglecting such variations would affect the accuracy of the forward problem and therefore the reconstruction of the source as well.

Nonetheless, in order to gain a deeper comprehension of the problem a rigorous mathematical analysis is essential providing a vital step towards the recognition of the underlying phenomena as well as identifying the limitations of the developed algorithms. The forward EEG problem has been extensively scrutinized for over sixty years since Wilson and Bayley [14] attempted to quantify the interplay between neuronal activity and the potentials they generate at the scalp. Apparently, for realistic head shapes the problem can only be solved numerically. Notwithstanding, the development of analytical algorithms in the event where the surface deviates from geometries which permit the installation of closed form solutions, such as the sphere or the ellipsoid, can be attempted 
in the framework of perturbation methods $[15,16]$. A first step towards understanding the contribution of surface deformations on the forward problem has been taken by Nolte and Curio [17]. The aforementioned authors, by realizing a perturbation technique different than the one displayed in the sequel, exploit the fact that if the shape of the head slightly varies from the sphere, one can accurately express the surface potential as a linear combination of spherical harmonics using only a small number of coefficients. Their analytical solution, derived utilizing Geselowitz's integral formula [18], does not involve a perturbation parameter in the usual sense.

The present paper pursues a similar perspective, that is, approximating the human brain by a homogeneous spherical conductor. Next, with the aid of explicit mathematical relations the surface of the model is distorted. First, we investigate the effect of surface deformations by localized impact on the forward EEG problem. In general, head injuries show a high incidence rate followed by devastating neurological outcome [19]. On the other hand, anthropometric analysis has documented a difference in the form of the human head depending on gender and ethnicity [20,21]. These differences on the forward EEG problem are examined in the sequel. To the authors' knowledge no data exists regarding the extent of closed head injuries (CHI) or in that manner geometric variations of the head in general. Under these circumstances, the deformations under considerations are considered small. Compared to the model by Nolte and Curio, the explicit solution presented incorporates a perturbation parameter $\epsilon$ which allows, independently of the chosen surface, an in-deep examination of the effect of the extent of surface deformations on the scalp potential. As a result, the firstorder correction provides a criterion in order to validate the significance of head shape deformations on EEG recordings.

The paper is structured as follows. Section 2 presents the mathematical background leading to closed form solutions for the forward EEG problem in spherical coordinates. The necessary adjustments which have to be made in order to obtain explicit solutions for a deformed spherical head are laid down in Section 3. In both sections, effort has been made to keep the mathematical display to a minimum, featuring only key relations. The interested reader will find the required details in order to obtain these expressions in the appendices. Finally, Section 4 is devoted to the numerical implementation and interpretation of the derived results whereas the Discussion summarizes the presented method and findings.

\section{Mathematical Formulation of the Forward Electroencephalographic Problem for a Spherical Conductor}

Presuppose a spherical homogeneous conductor with radius $a$ and conductivity $\sigma$ occupying a finite domain $D \subset \mathbb{R}^{3}$ confined by a smooth boundary $\partial D$ serving as an approximation for the brain. Identify by $D^{c}$ the exterior domain to $D$ where the conductivity is zero.

Activation of a localized region in the brain triggers a primary neuronal current $\mathbf{J}^{p}$ generating an electric field $\mathbf{E}$ as well as a magnetic induction field $\mathbf{B}$, respectively. In the case where the neuronal current is represented by a single equivalent dipole at the point $\mathbf{r}_{0}$ with moment $\mathbf{Q}$, then $\mathbf{J}^{p}=$ $\mathbf{Q} \delta\left(\mathbf{r}-\mathbf{r}_{0}\right), \delta$ denoting the Dirac measure.

Plonsey and Heppner [22] demonstrated that the electromagnetic activity of the brain is governed by the quasistatic theory of Maxwell's equations, namely,

$$
\begin{gathered}
\nabla \times \mathbf{E}=\mathbf{0}, \\
\nabla \times \mathbf{B}=\mu_{0}\left(\mathbf{J}^{p}+\sigma \mathbf{E}\right), \\
\nabla \cdot \mathbf{B}=0,
\end{gathered}
$$

where the magnetic permeability $\mu_{0}$ is assumed to be constant everywhere in $\mathbb{R}^{3}$.

Equation (1) allows the introduction of an electric potential $u$ such that

$$
\mathbf{E}=-\nabla u
$$

Moreover, by taking the divergence of (2), we immediately conclude that the interior electric potential $u^{-}$solves the following Neumann boundary value problem in $D$ :

$$
\begin{gathered}
\Delta u^{-}\left(\mathbf{r}, \mathbf{r}_{0}\right)=\frac{1}{\sigma} \mathbf{Q} \cdot \nabla \delta\left(\mathbf{r}-\mathbf{r}_{0}\right), \quad r<a, \\
\frac{\partial}{\partial r} u^{-}\left(\mathbf{r}, \mathbf{r}_{0}\right)=0, \quad r=a,
\end{gathered}
$$

where the operators $\Delta$ and $\nabla$ act on the point of observation r.

Once the above problem is solved, knowledge of the solution $u^{-}$leads to the exterior electric potential $u^{+}$satisfying the Dirichlet problem

$$
\begin{gathered}
\Delta u^{+}\left(\mathbf{r}, \mathbf{r}_{0}\right)=0, \quad r>a, \\
u^{+}\left(\mathbf{r}, \mathbf{r}_{0}\right)=u^{-}\left(\mathbf{r}, \mathbf{r}_{0}\right), \quad r=a, \\
u^{+}\left(\mathbf{r}, \mathbf{r}_{0}\right)=\mathcal{O}\left(\frac{1}{r^{2}}\right), \quad r \longrightarrow \infty
\end{gathered}
$$

According to the right-hand side of (5) it is evident that each nontrivial solution of the EEG problem is generated by the source activity, which we rewrite in the form

$$
\mathbf{Q} \cdot \nabla_{\mathbf{r}} \delta\left(\mathbf{r}-\mathbf{r}_{0}\right)=-\mathbf{Q} \cdot \nabla_{\mathbf{r}_{0}} \delta\left(\mathbf{r}-\mathbf{r}_{0}\right)
$$

and therefore it suffices to analyze the action of the directional derivative $\mathbf{Q} \cdot \nabla_{\mathbf{r}_{0}}$. Further, it is eminent that the action of this specific directional derivative on the field of a monopole generates the field of a dipole [23]. Hence, the general problem can be solved by considering a monopole source while the corresponding solution for a dipole source is easily evaluated by acting with the directional derivative correlated to the pair $\left\{\mathbf{r}_{0}, \mathbf{Q}\right\}$. For this purpose we introduce the potential $v$ associated with a unit monopole source located at $\mathbf{r}_{0}$ as

$$
u\left(\mathbf{r}, \mathbf{r}_{0}\right)=\frac{1}{\sigma} \mathbf{Q} \cdot \nabla_{\mathbf{r}_{0}} v\left(\mathbf{r}, \mathbf{r}_{0}\right) .
$$


Under the above assumption, boundary-value problems (5), (6), and (7) simplify as follows:

$$
\begin{gathered}
\Delta v^{-}\left(\mathbf{r}, \mathbf{r}_{0}\right)=-\delta\left(\mathbf{r}-\mathbf{r}_{0}\right), \quad r<a, \\
\frac{\partial}{\partial r} v^{-}\left(\mathbf{r}, \mathbf{r}_{0}\right)=0, \quad r=a,
\end{gathered}
$$

as well as

$$
\begin{gathered}
\Delta v^{+}\left(\mathbf{r}, \mathbf{r}_{0}\right)=0, \quad r>a, \\
v^{+}\left(\mathbf{r}, \mathbf{r}_{0}\right)=v^{-}\left(\mathbf{r}, \mathbf{r}_{0}\right), \quad r=a, \\
v^{+}\left(\mathbf{r}, \mathbf{r}_{0}\right)=\mathcal{O}\left(\frac{1}{r^{2}}\right), \quad \mathbf{r} \longrightarrow \infty .
\end{gathered}
$$

Employing analytic techniques (see $[3,4]$ for details), it is not hard to show that the solution regarding (5) and (6) is

$$
u^{-}\left(\mathbf{r}, \mathbf{r}_{0}\right)=\frac{\mathbf{Q}}{4 \pi \sigma} \cdot\left(\frac{\mathbf{P}}{P^{3}}+\frac{a}{r} \frac{\mathbf{R}}{R^{3}}+\frac{1}{a R} \frac{\mathbf{r} R+r \mathbf{R}}{r R+\mathbf{r} \cdot \mathbf{R}}\right)
$$

whereas the solution concerning (7) is

$$
u^{+}\left(\mathbf{r}, \mathbf{r}_{0}\right)=\frac{\mathbf{Q}}{4 \pi \sigma} \cdot\left(\frac{2 \mathbf{P}}{P^{3}}+\frac{1}{r P} \frac{r \mathbf{P}+\mathbf{r} P}{r P+\mathbf{r} \cdot \mathbf{P}}\right)
$$

where

$$
\mathbf{P}=\mathbf{r}-\mathbf{r}_{0}, \quad \mathbf{R}=\frac{a^{2}}{r^{2}} \mathbf{r}-\mathbf{r}_{0} .
$$

On the other hand, the corresponding surface values are easily evaluated to be

$$
\begin{aligned}
u\left(a \widehat{\mathbf{r}}, \mathbf{r}_{0}\right)=\frac{\mathbf{Q}}{4 \pi \sigma} \cdot( & 2 \frac{a \widehat{\mathbf{r}}-\mathbf{r}_{0}}{\left|a \widehat{\mathbf{r}}-\mathbf{r}_{0}\right|^{3}} \\
& \left.+\frac{1}{a\left|a \widehat{\mathbf{r}}-\mathbf{r}_{0}\right|} \frac{\left|a \widehat{\mathbf{r}}-\mathbf{r}_{0}\right| \widehat{\mathbf{r}}+\left(a \widehat{\mathbf{r}}-\mathbf{r}_{0}\right)}{\left|\mathbf{r}_{0}\right|+\widehat{\mathbf{r}} \cdot\left(a \widehat{\mathbf{r}}-\mathbf{r}_{0}\right)}\right) .
\end{aligned}
$$

\section{Contribution of Surface Deformations on the Forward EEG Problem}

The present section provides the analysis leading to closed form solutions for the forward EEG problem implicating deformations of the heads surface by explicit relations. To this end, replace the conductor considered in Section 2 by a locally deformed spherical conductor, depicted in Figure 1, occupying a finite domain $D^{p} \subset \mathbb{R}^{3}$ confined by a smooth boundary $\partial D^{p}$, sharing the same physical characteristics as in the unperturbed case. The interior boundary value problem (BVP) (10), (11) together with the exterior BVP (12)-(14) described in Section 2 can now not be solved analytically in $D^{p}$ or in $D^{p, c}=\mathbb{R}^{3} / D^{p} \cup \partial D^{p}$, respectively.

However, it is possible to transform the corresponding BVPs regarding the nonsymmetrical "spherical" conductor to approximate problems with reference to the symmetrical case. To this end, assume $\mathbf{r}^{p}$ to be the distance of any point

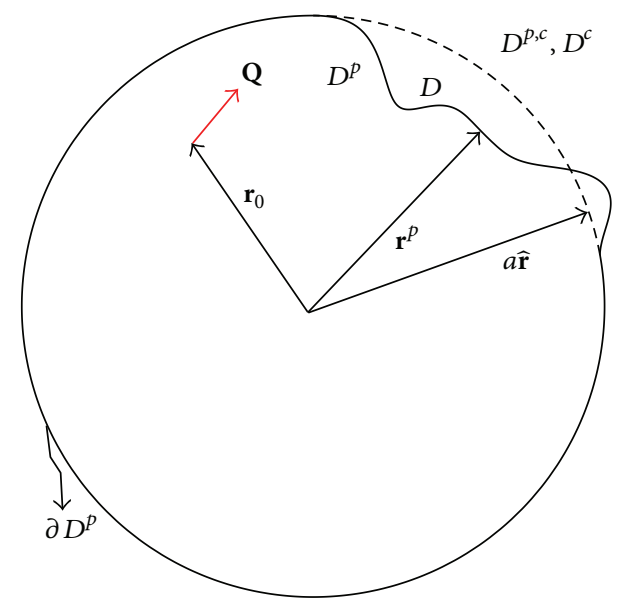

FIgURE 1: A locally deformed homogeneous sphere (continuous line) of radius $r^{p}(\theta, \phi ; \epsilon)$ merged with the kindred nondeformed (dashed line) sphere. For domains in between the nondeformed and deformed conductor, depending on if $r^{p}<a$ or $r^{p}>a, D$ coincides with $D^{p, c}$ or $D^{p}=D^{c}$, respectively. Obviously, for nondeformed regions $\epsilon=0$ and $r^{p}(\theta, \phi ; 0)=a$.

lying on the boundary $\partial D^{p}$. Apparently, the Euclidean norm of $\mathbf{r}^{p}$ can be expressed in the form $r^{p}=a+\left(r^{p}-a\right)$, where the difference $r^{p}-a$ provides the means of measuring the departure between the unperturbed boundary $\partial D$ and the perturbed one $\partial D^{p}$. Introducing additionally a parameter $\epsilon$ we obtain

$$
r^{P}(\theta, \phi ; \epsilon)=a+\epsilon f(\theta, \phi),
$$

where the known function $f(\theta, \phi)$ denotes the aforementioned difference carrying dimensions of length. Clearly, in the case where $\epsilon$ vanishes, the unperturbed case described earlier is recovered.

In the sequel, a Poincaré type solution of the form

$$
v\left(\mathbf{r}^{p}\right)=\sum_{n=0}^{\infty} \epsilon^{n} v_{n}(\mathbf{r})
$$

is sought, where the perturbation parameter $\epsilon$ is considered to be close to zero and the coefficients $v_{n}$ are independent of the perturbation parameter. Note that the left-hand side of (20) refers to the point $\mathbf{r}^{p} \in D^{p}$, expressed as $\mathbf{r}^{p}=\mathbf{r}+\epsilon f(\theta, \phi) \widehat{\mathbf{r}}$, whereas the right-hand side of (20) refers to the point $\mathbf{r} \in D$.

The interior and exterior electric potentials are then straightforwardly evaluated by replacing (20) into the corresponding partial differential equations (PDE) (10) and (12), respectively. Specifically, for the interior potential $v^{-}\left(\mathbf{r}^{p}, \mathbf{r}_{0}\right)$ this procedure returns, after collecting coefficients of $\epsilon$, the following infinite system:

$$
\begin{gathered}
\Delta v_{0}^{-}\left(\mathbf{r}, \mathbf{r}_{0}\right)=-\delta\left(\mathbf{r}-\mathbf{r}_{0}\right), \\
\Delta v_{n}^{-}\left(\mathbf{r}, \mathbf{r}_{0}\right)=0, \quad n \geq 1 .
\end{gathered}
$$

Similar, the exterior electric potential $v^{+}\left(\mathbf{r}^{p}, \mathbf{r}_{0}\right)$ is provided by the system

$$
\Delta v_{n}^{+}\left(\mathbf{r}, \mathbf{r}_{0}\right)=0, \quad n \geq 0 .
$$


The latter equations have to be accompanied by matching boundary conditions (see Appendix A). Apparently, the above analysis assumes the dipole source to be consistently located inside the perturbed domain $r^{p}<a$.

3.1. The Electric Potential. In the linear regime, the interior electric potential is provided computing the first two coefficients of (20), namely, $v_{0}$ and $v_{1}$. The zeroth-order correction $v_{0}$ is retrieved through (21) combined with (A.3) for $n=0$ and the solution is given by (15).

On the other hand, the first-order correction $v_{1}$ is determined solving the following BVP:

$$
\begin{gathered}
\Delta v_{1}^{-}\left(\mathbf{r}, \mathbf{r}_{0}\right)=0, \quad r<a \\
\frac{\partial}{\partial r} v_{1}^{-}\left(\mathbf{r}, \mathbf{r}_{0}\right)=\frac{1}{a^{2}}\left(\frac{\partial f}{\partial \theta} \frac{\partial v_{0}^{-}}{\partial \theta}+\frac{1}{\sin ^{2} \theta} \frac{\partial f}{\partial \phi} \frac{\partial v_{0}^{-}}{\partial \phi}\right), \quad r=a
\end{gathered}
$$

subject to

$$
\oint_{\|\mathbf{r}\|=a} \nabla_{\mathbf{r}} f(\widehat{\mathbf{r}}) \cdot \nabla_{\mathbf{r}} v_{0}\left(\mathbf{r}, \mathbf{r}_{0}\right) \mathrm{d} \Omega(\widehat{\mathbf{r}})=0,
$$

where $\mathrm{d} \Omega(\widehat{\mathbf{r}})=\sin \theta \mathrm{d} \theta \mathrm{d} \phi$ denotes the solid angle element.

After tedious calculations (see Appendix B for details), the first-order correction $v_{1}$ can be expressed as

$$
\begin{aligned}
v_{1}\left(\mathbf{r}, \mathbf{r}_{0}\right)= & \sum_{p=1}^{\infty} \sqrt{\frac{2 p+1}{4 \pi}} \mathscr{B}_{p}^{0}\left(r, \mathbf{r}_{0}\right) r^{p} P_{p}(\cos \theta) \\
& +2 \operatorname{Re} \sum_{p=1}^{\infty} \sum_{q=1}^{p} \mathscr{B}_{p}^{q}\left(r, \mathbf{r}_{0}\right) r^{p} Y_{p}^{q}(\widehat{\mathbf{r}}),
\end{aligned}
$$

where Re is the real part of the subsequent expansion whereas the corresponding coefficients are given as

$$
\begin{aligned}
& \mathscr{B}_{p}^{q}=\frac{1}{p a^{p+1}} \mathscr{D}_{p}^{q}+\frac{(-1)^{q}}{p a^{p+1}} \\
& \times\left[\sum_{n=p-2}^{\infty} \sum_{k=1}^{n} \sum_{m=-k}^{k} \frac{r_{0}^{k} \bar{Y}_{k}^{m}\left(\widehat{\mathbf{r}}_{0}\right)}{a^{k+1}} \beta_{s}^{q-m} j_{k+1}^{m} K_{k+1, s, p}\right. \\
& \times\left(\begin{array}{ccc}
k+1 & s & p \\
0 & 0 & 0
\end{array}\right)\left(\begin{array}{ccc}
k+1 & s & p \\
m & q-m & -q
\end{array}\right) \\
& -\sum_{n=p}^{\infty} \sum_{k=1}^{n} \sum_{m=-k}^{k} \frac{k+1}{k} \frac{r_{0}^{k} \bar{Y}_{k}^{m}\left(\widehat{\mathbf{r}}_{0}\right)}{a^{k+1}} \beta_{s}^{q-m} j_{k}^{m} K_{k-1, s, p} \\
& \times\left(\begin{array}{ccc}
k-1 & s & p \\
0 & 0 & 0
\end{array}\right)\left(\begin{array}{ccc}
k-1 & s & p \\
m & q-m & -q
\end{array}\right) \\
& +i \sum_{n=p-1}^{\infty} \sum_{k=1}^{n} \sum_{m=-k}^{k} \frac{m}{k} \frac{r_{0}^{k} \bar{Y}_{k}^{m}\left(\widehat{\mathbf{r}}_{0}\right)}{a^{k+1}} \gamma_{s}^{q-m} K_{k, s, p} \\
& \left.\times\left(\begin{array}{ccc}
k & s & p \\
0 & 0 & 0
\end{array}\right)\left(\begin{array}{ccc}
k & s & p \\
m & q-m & -q
\end{array}\right)\right] \quad p \geq 1,
\end{aligned}
$$

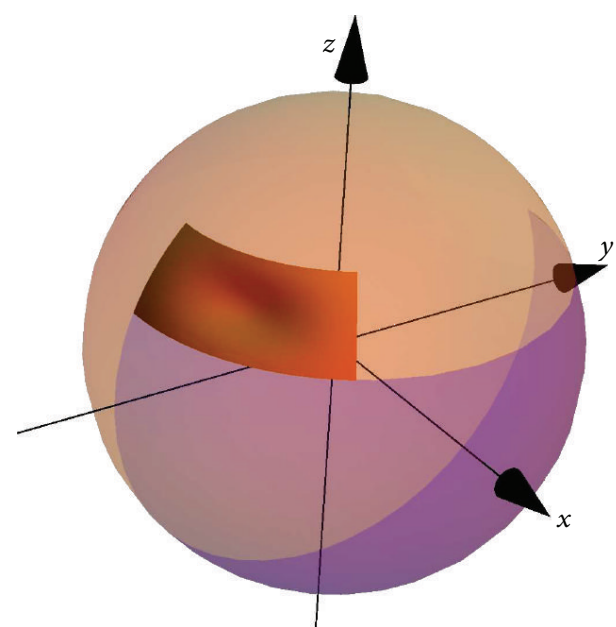

FIGURE 2: A nonpenetrating head injury in form of a sunken area (orange) situated at the fore part of the head, just above the facial region (purple) occupying $2.7 \%$ of the total area. The extent of the injury as displayed is for illustrative purpose only.

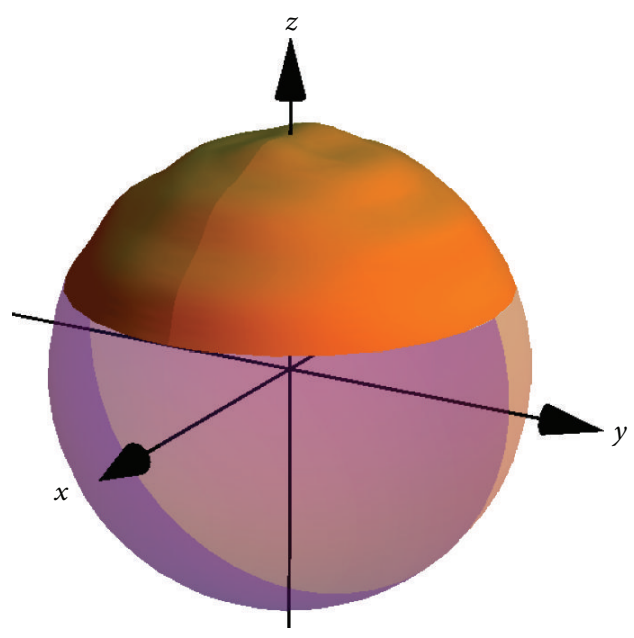

FIGURE 3: Deviation of the head shape for the upper part of the human cranium consisting of cavities and bulges. The deformed region holds $31.7 \%$ of the entire area. The extent of the deformation as displayed is for illustrative purpose only.

given that $s=n-k+1$ and

$$
\begin{aligned}
\mathscr{D}_{p}^{q}= & \frac{\beta_{0}^{0}}{\sqrt{4 \pi}}\left(\zeta_{p}-\frac{r_{0}^{p+1} \bar{Y}_{p+1}^{q}\left(\widehat{\mathbf{r}}_{0}\right)}{a^{p+2}} \frac{p+2}{p+1} j_{p+1}^{q}\right) \\
& +i q \frac{\gamma_{0}^{0}}{\sqrt{4 \pi}} \frac{r_{0}^{p} \bar{Y}_{p}^{q}\left(\widehat{\mathbf{r}}_{0}\right)}{p a^{p+1}},
\end{aligned}
$$

with

$$
\zeta_{1}=0, \quad \zeta_{p}=\frac{r_{0}^{p-1} \bar{Y}_{p-1}^{q}\left(\widehat{\mathbf{r}}_{0}\right)}{a^{p}} j_{p}^{q}, \quad p \geq 2
$$

Whereas the coefficients $\beta_{s}^{\ell}$ and $\gamma_{s}^{\ell}$ are provided via (B.8) and (B.9), respectively, incorporating information about 
(1) $\theta$

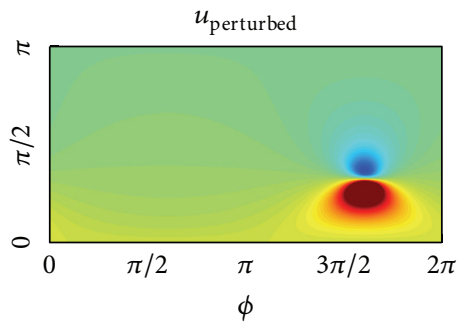

(a)

(2) $\theta$

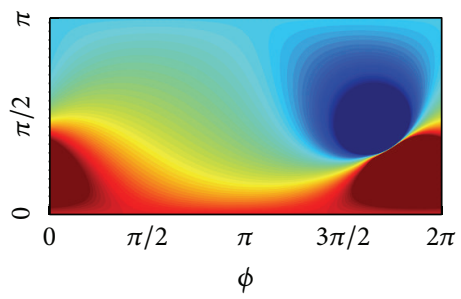

(d)

(3) $\theta$

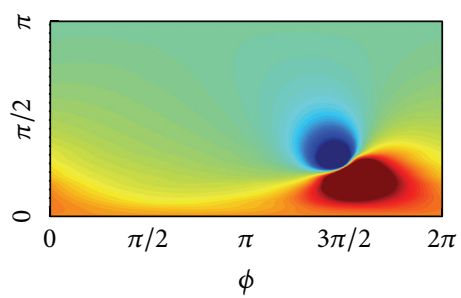

(g)

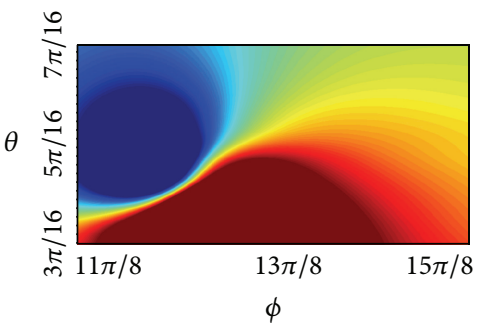

(j)

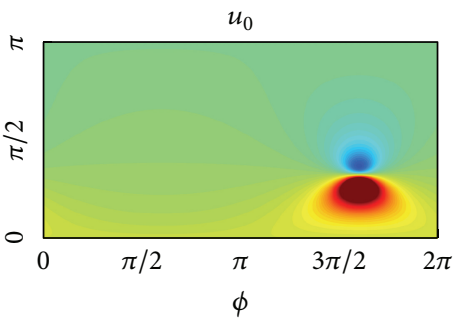

(b)

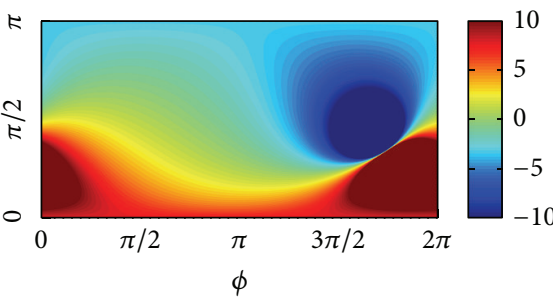

(e)

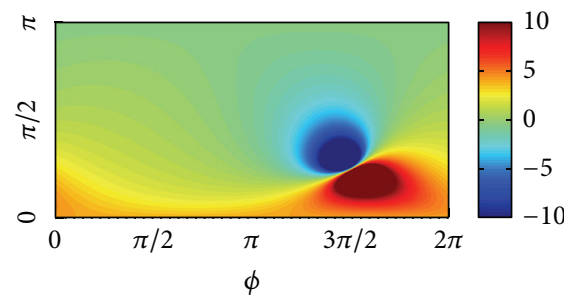

(h)

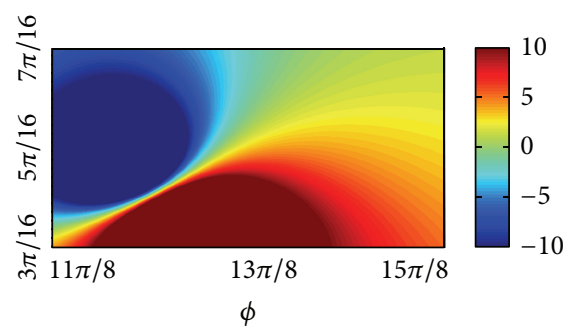

(k)
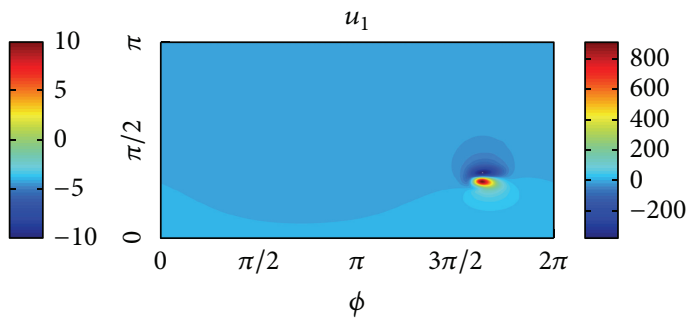

(c)

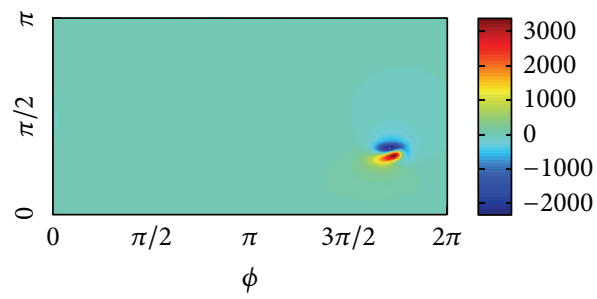

(f)

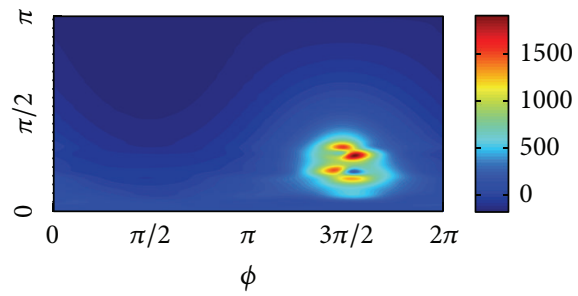

(i)

FIGURE 4: Simulated EEG recording ( $\mathrm{mV} \mathrm{s}$ ) originating from a spherical homogeneous conductor containing a small cavity (left column) as described in Section 4.1 in the case of neuronal excitation beneath the injury. Each row represents a specific dipole arrangement, namely, (1) positioned at $r_{0}=0.06 \mathrm{~m}$ and $\theta_{0}=0.29 \pi, \phi_{0}=1.6 \pi$ with moments $\mathbf{Q}=(0,0,1) \times 10^{-5} \mathrm{Cm}$; (2) at $r_{0}=0.06 \mathrm{~m}, \theta_{0}=0.33 \pi, \phi_{0}=1.75 \pi$, and $\mathbf{Q}=(2,3,5) \times 10^{-5} \mathrm{Cm}$; (3) situated at $r_{0}=0.06 \mathrm{~m}, \theta_{0}=0.25 \pi, \phi_{0}=1.5 \pi$, and $\mathbf{Q}=(1,1,1) \times 10^{-5} \mathrm{Cm}$. The corresponding recording concerning the unperturbed instance $u_{0}$ is shown in the middle column for the analogous dipole locations and moments, whereas (c), (f), and (i) specify the contribution of the first-order correction $u_{1}$ on $u_{\text {perturbed }}=u\left(\mathbf{r}^{p}\right)$ as expressed by (32) for $\epsilon=0.004$. On the other hand, Figures $(\mathrm{j})$ and $(\mathrm{k})$ provide a closer visual examination regarding the effect of the perturbation in the neighborhood of the excitation.

the deformation, namely, the known function $f(\theta, \phi)$, the coefficients $K$ are given by (B.14).

In accord with previous computations, the exterior electric potential $v^{+}\left(\mathbf{r}^{p}\right)$ is evaluated in a similar fashion.

\section{Results}

In order to visually translate the mathematics in play, a spherical head model is introduced, consisting of two major compartments: (i) the facial region located between $\theta \in$ $[3 \pi / 8, \pi]$ and $\phi \in[3 \pi / 2, \pi / 2]$, indicated with purple color in
Figures 2 and 3 included solely for the purpose of reference; (ii) the deformed partition indicated with orange color. The surface deformations presented are generated by means of the function

$$
f(\theta, \phi)=(\sin \kappa \theta)^{\nu}(\cos \lambda \phi)^{\mu}
$$

For the simulations, the following values for the parameters have been adopted. A radius of $0.071 \mathrm{~m}$ has been utilized corresponding to an average volume of the brain of $1.5 \mathrm{~L}$, whereas the conductivity of the brain equals $0.30 \mathrm{~S} / \mathrm{m}[24,25]$. 
TABLE 1: Introduced errors neglecting local distortions for selected positions and specific values of the perturbation parameter $\epsilon$ on EEG measurements, as displayed in Figure 5.

\begin{tabular}{|c|c|c|c|c|c|}
\hline \multirow[b]{2}{*}{$\theta_{0}, \phi_{0}$} & \multirow[b]{2}{*}{$\epsilon$} & \multicolumn{2}{|c|}{ Position A } & \multicolumn{2}{|c|}{ Position B } \\
\hline & & $\begin{array}{c}u \\
(\mathrm{mV} \mathrm{s})\end{array}$ & $\begin{array}{c}\text { Error } \\
\%\end{array}$ & $\begin{array}{c}u \\
(\mathrm{mV} \mathrm{s})\end{array}$ & $\begin{array}{c}\text { Error } \\
\%\end{array}$ \\
\hline \multirow{6}{*}{$0.3 \pi, 1.75 \pi$} & 0 & -0.1739 & & -0.6051 & \\
\hline & 0.002 & -0.1727 & 0.69 & -0.6087 & 0.59 \\
\hline & 0.004 & -0.1716 & 1.32 & -0.6123 & 1.19 \\
\hline & 0.006 & -0.1704 & 2.01 & -0.6159 & 1.78 \\
\hline & 0.008 & -0.1692 & 2.70 & -0.6195 & 2.38 \\
\hline & 0.010 & -0.1680 & 3.39 & -0.6232 & 2.99 \\
\hline \multirow{6}{*}{$0.33 \pi, 1.75 \pi$} & 0 & -1.559 & & -4.333 & \\
\hline & 0.002 & -1.542 & 1.09 & -4.343 & 0.23 \\
\hline & 0.004 & -1.526 & 2.12 & -4.353 & 0.46 \\
\hline & 0.006 & -1.510 & 3.14 & -4.362 & 0.67 \\
\hline & 0.008 & -1.494 & 4.16 & -4.372 & 0.90 \\
\hline & 0.010 & -1.478 & 5.19 & -4.382 & 1.13 \\
\hline \multirow{6}{*}{$0.32 \pi, 1.7 \pi$} & 0 & -0.6896 & & -1.299 & \\
\hline & 0.002 & -0.6757 & 2.02 & -1.290 & 0.69 \\
\hline & 0.004 & -0.6618 & 4.03 & -1.281 & 1.38 \\
\hline & 0.006 & -0.6478 & 6.06 & -1.272 & 2.08 \\
\hline & 0.008 & -0.6339 & 8.08 & -1.263 & 2.77 \\
\hline & 0.010 & -0.6200 & 10.1 & -1.254 & 3.46 \\
\hline
\end{tabular}

Moreover, only cortical neuronal excitations are examined at a distance of $r_{0}=0.06 \mathrm{~m}$ and various values of $\theta_{0}$ and $\phi_{0}$ depending on (26). Further, the present study adapts values for the moment of the dipoles in the order of $10^{-5} \mathrm{Cm}$, ensuring that the measured potential ranges between a few $\mathrm{mV}$ s up to about $100 \mathrm{mV} \mathrm{s}$ [26]. The following configurations have been implemented for the simulation of the reference brain activity: (1) a dipole parallel to the $z$-axis with moments $\mathbf{Q}=(0,0,1) \times 10^{-5} \mathrm{Cm},(2)$ a dipole with equal moments $\mathbf{Q}=(1,1,1) \times 10^{-5} \mathrm{Cm}$, and (3) A dipole arbitrary oriented and moments $\mathbf{Q}=(2,3,5) \times 10^{-5} \mathrm{Cm}$. However, the influence of the dipoles orientation on the overall investigation in the framework of the presented analysis is nominal. We already mentioned that to the authors' knowledge no data regarding the extent of closed head injuries or for geometric variations of the head in general exist. Under these circumstances, a value of 0.004 has been introduced, equivalent to a maximum depth for cavities (or maximum height for bulges) of four millimeters.

4.1. Local Surface Distortions. For the first example, we consider a closed, nonpenetrating head injury in form of a blow to the head right above the facial region, depicted in Figure 2, represented by (31) with parameters $(\kappa, \lambda, \nu, \mu)=$ $(8,3,2,3)$ defined in the intervals $\theta \in[\pi / 4,3 \pi / 8]$ and $\phi \in[3 \pi / 2,11 \pi / 6]$. These kinds of injuries are commonly associated with home and occupational accidents as well as motor vehicle traffic collisions causing traumatic brain injuries (TBI) disrupting the normal function of the brain.

The calculated potential on the surface of the conductor (unperturbed and perturbed likewise) largely ranges between approximately $-50 \mathrm{mV} \mathrm{s}$ and $50 \mathrm{mV} \mathrm{s}$, the extrema situated in the neighborhood of the cortical excitation. For areas at a short radius to the neuronal activity the recordings decrease/increase almost instantly to one-fifth of the highest/lowest values, respectively. On the other hand, the values of the potential vary roughly one to two orders in magnitude for regions at a distance of the dipoles location. Figure 4 provides an overview whereby above-presented surface deformation influences the EEG measurements generated by a dipole positioned exactly under the injury. The spherical homogeneous conductor without any deformation present (middle column) is compared to the perturbed case (left column). In order to display small changes of the surface potential the corresponding values have been downscaled. It is observed that adjusting a small area (approximately 3\%) of the surface the EEG recordings as a whole are minimally affected. Specifically, a moderate alteration about $\pm 0.5 \mathrm{mV} \mathrm{s}$ has been found in the close vicinity of the dipole whereas in the remaining areas the values stay relatively the same $( \pm 0.05 \mathrm{mV} \mathrm{s})$. However, these differences intensify with the severity of the trauma (shape and depth). On the other hand, the potential recordings for active regions away from the distortion site are not affected.

Figure 5 provides a point specific observation of the electric potential on the deformed surface and surroundings. For neuronal activity adjacent to the surface, simulated by dipoles located at $r_{0}=0.06 \mathrm{~m}$, and specific values $\theta_{0}, \phi_{0}$ dictated by (26), Figures 5(a)-5(f) show collectively how the potential alters on the rays $\theta=0.5 \pi, \phi=\pi$ (position A) and $\theta=0.75 \pi, \phi=1.25 \pi$ (position B), respectively, as well as the differences detected for four distinct values 
TABLE 2: Introduced errors neglecting geometric variations of the upper part of the head for selected positions and specific values of the perturbation parameter $\epsilon$ on EEG measurements, as displayed in Figure 7.

\begin{tabular}{|c|c|c|c|c|c|}
\hline \multirow{3}{*}{$\theta_{0}, \phi_{0}$} & \multirow{3}{*}{$\epsilon$} & \multicolumn{2}{|c|}{ Position A } & \multicolumn{2}{|c|}{ Position B } \\
\hline & & $u$ & Error & $u$ & Error \\
\hline & & $(\mathrm{mV} \mathrm{s})$ & $\%$ & $(\mathrm{mV} \mathrm{s})$ & $\%$ \\
\hline \multirow{6}{*}{$0.29 \pi, 1.65 \pi$} & 0 & -0.2117 & & -0.7123 & \\
\hline & 0.002 & -0.2043 & 3.49 & -0.7154 & 0.43 \\
\hline & 0.004 & -0.1969 & 6.99 & -0.7184 & 0.85 \\
\hline & 0.006 & -0.1894 & 10.53 & -0.7214 & 1.28 \\
\hline & 0.008 & -0.1820 & 14.03 & -0.7244 & 1.70 \\
\hline & 0.010 & -0.1746 & 17.52 & -0.7275 & 2.13 \\
\hline \multirow{6}{*}{$0.17 \pi, 1.55 \pi$} & 0 & -3.003 & & -4.563 & \\
\hline & 0.002 & -3.086 & 2.76 & -4.539 & 0.52 \\
\hline & 0.004 & -3.168 & 5.49 & -4.515 & 1.05 \\
\hline & 0.006 & -3.251 & 8.26 & -4.491 & 1.58 \\
\hline & 0.008 & -3.333 & 10.99 & -4.467 & 2.10 \\
\hline & 0.010 & -3.416 & 13.75 & -4.443 & 2.63 \\
\hline \multirow{6}{*}{$0.32 \pi, 1.55 \pi$} & 0 & -0.7185 & & -1.416 & \\
\hline & 0.002 & -0.6819 & 5.09 & -1.420 & 0.28 \\
\hline & 0.004 & -0.6453 & 10.2 & -1.423 & 0.49 \\
\hline & 0.006 & -0.6087 & 15.3 & -1.427 & 0.77 \\
\hline & 0.008 & -0.5721 & 20.4 & -1.431 & 1.06 \\
\hline & 0.010 & -0.5355 & 25.5 & -1.434 & 1.27 \\
\hline
\end{tabular}

of the perturbation parameter $\epsilon$. Each row corresponds to a particular orientation of the dipole, namely, from top to bottom; $\mathbf{Q}=(0,0,1) \times 10^{-5} \mathrm{Cm}, \mathbf{Q}=(1,1,1) \times 10^{-5} \mathrm{Cm}$, and $\mathbf{Q}=(2,3,5) \times 10^{-5}$, respectively. We observe that the errors made neglecting regional surface deformations for various $\epsilon$ depending on the region of activity and the point of surveillance are in general small, except peculiar situations (see, e.g., Figure 5(e)). These instances are summarized in Table 1.

Analogous conclusions uphold for similar injuries or traumas on the back or side of the head, materializing after falls or physical assaults.

\subsection{Deformation of the Upper Part of the Human Cranium.} In what follows, we analyze the instance where the upper compartment of the human skull exhibits irregularities in appearance. The examined situation is of particular interest bearing in mind that, first of all, the top part of the head consists of the main measurement site for EEG recordings and, secondly, human head shapes vary considerably among individuals.

The perturbed surface, depicted in Figure 3, is constructed via (31) with parameters $(\kappa, \lambda, \nu, \mu)=(8,2,5,1)$ defined in the intervals $\theta \in[0,3 \pi / 8]$ and $\phi \in(0,2 \pi]$.

The impact of such anatomical irregularities on Electroencephalographic measurements is investigated considering miscellaneous locations and configurations for the dipole. In all cases, the firing of neurons occurs in close range to the deformation site $\left(r_{0}=0.06 \mathrm{~m}\right)$. Depending on the dipoles position, the surface potential fluctuates largely between $-40 \mathrm{mV} \mathrm{s}$ and $60 \mathrm{mV} \mathrm{s}$. Again, the maximum and minimum values are found in the proximity of the active region. Similar to the aforementioned example, small differences in the magnitude of the surface potential are also observed affecting the entire spectrum of recordings. These ramifications are displayed in Figure 6 where the corresponding values have been one more time downscaled in order to highlight indicated aspects.

The electric potential immediate and including the perturbed surface at specific positions is shown in Figure 7. In particular, Figures $7(\mathrm{a})-7(\mathrm{f})$ provide insight into how the potential alters on the rays $(\theta, \phi)=(0.5 \pi, \pi)$ (left column) and $(\theta, \phi)=(0.75 \pi, 1.25 \pi)$ (right column) for distinct source locations and direction. Although it is observed that the presence of geometric variations modifies the recorded potential the errors introduced ignoring surface alterations of the head are, in general, minimal. However, depending on the "distortion" displayed by the upper compartment of the head combined with the position and strength of the dipole errors up to $25 \%$ could be observed (see Table 2 ). These possibilities can be detected with the assistance of the firstorder correction $u_{1}$ (see, e.g., the corresponding graph of $u_{1}$ displayed in Figure 6(f) where an extensive area with values about 200 to $300 \mathrm{mV}$ s exists).

\section{Discussion}

Introducing a homogeneous spherical model, we perform a first-order perturbation analysis in order to examine the influence of geometric variations present on the conductors surface on the forward EEG problem. The presented deviations describe two major cases: (1) localized acquired 


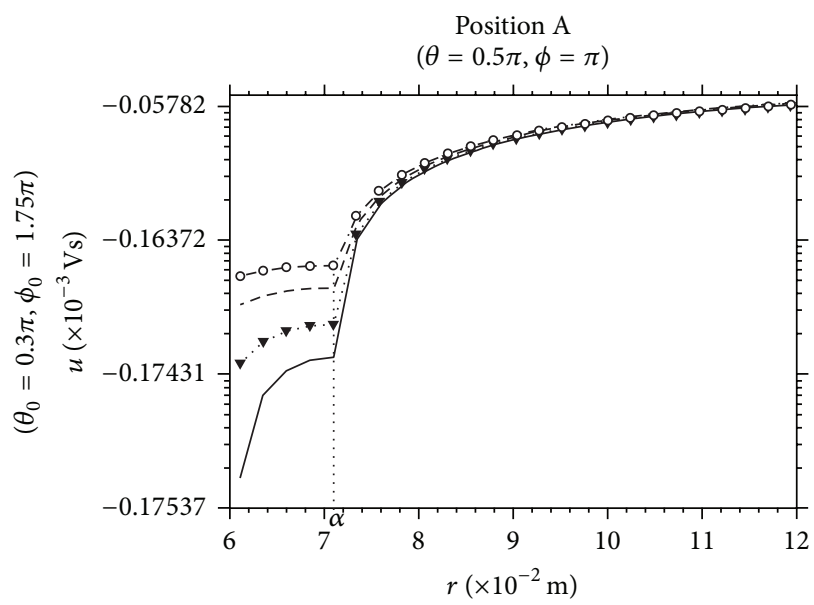

(a)

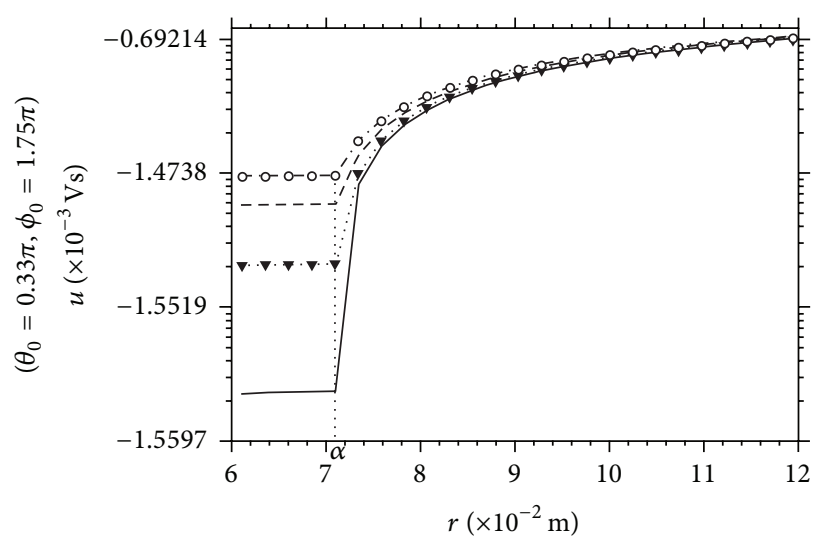

(c)

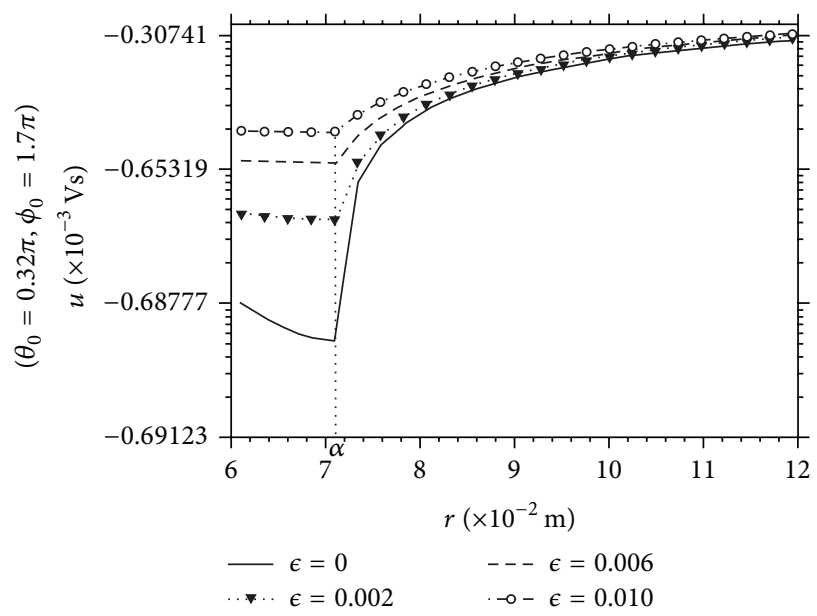

(e)

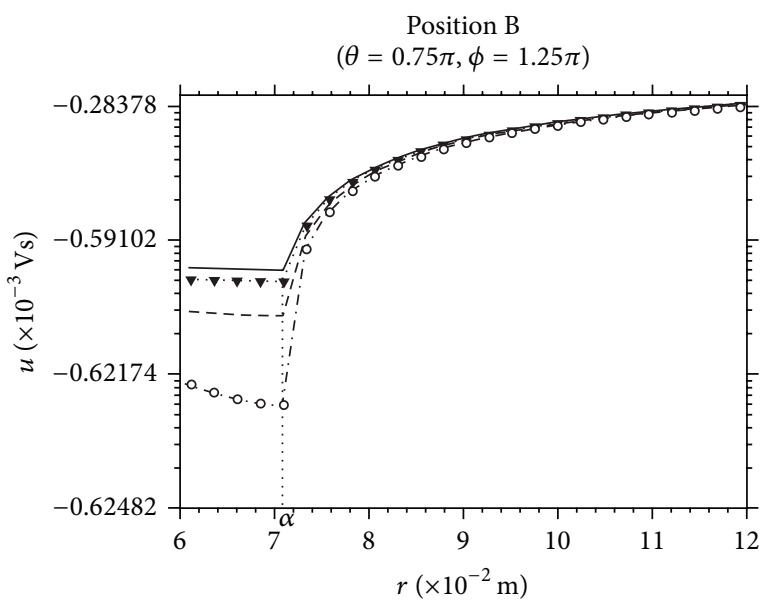

(b)

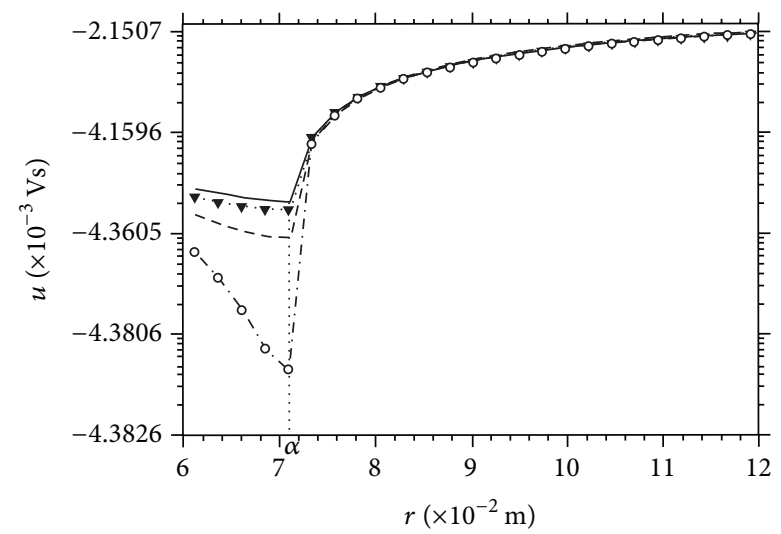

(d)

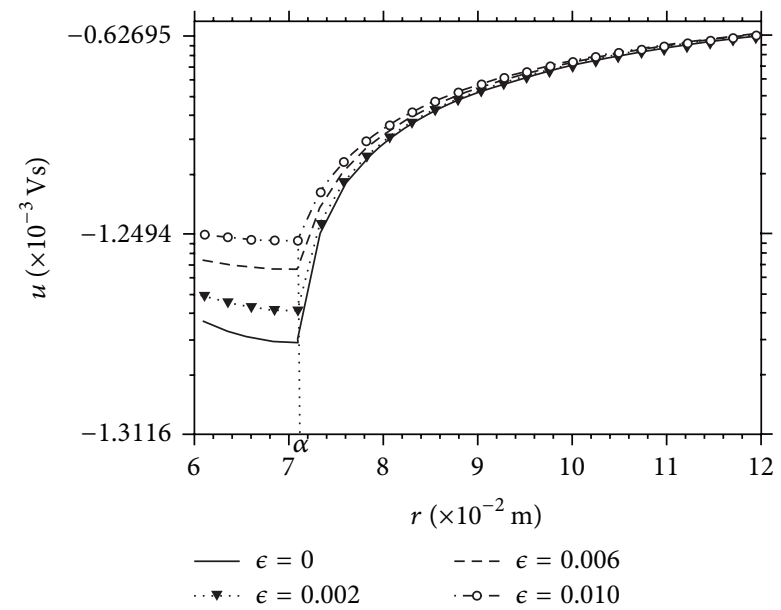

(f)

FIGURE 5: The electric potential on the surface $r^{p}(\theta, \phi ; \epsilon)$ and surroundings, along the rays $\theta=0.5 \pi, \phi=\pi$ (position A, left column) and $\theta=0.75 \pi, \phi=1.25 \pi$ (position $\mathrm{B}$, right column), respectively, for distinct values of the perturbation parameter $\epsilon$, depending on the severity of the nonpenetrating head wound as displayed in Figure 2. Each row represents a specific dipole arrangement, namely, (1) positioned at $r_{0}=0.06 \mathrm{~m}$ and $\theta_{0}=0.29 \pi, \phi_{0}=1.75 \pi$ with moments $\mathbf{Q}=(0,0,1) \times 10^{-5} \mathrm{Cm}$; (2) at $r_{0}=0.06 \mathrm{~m}, \theta_{0}=0.33 \pi, \phi_{0}=1.75 \pi$, and $\mathbf{Q}=(2,3,5) \times 10^{-5} \mathrm{Cm}$; (3) situated at $r_{0}=0.06 \mathrm{~m}, \theta_{0}=0.32 \pi, \phi_{0}=1.7 \pi$, and $\mathbf{Q}=(1,1,1) \times 10^{-5} \mathrm{Cm}$. 


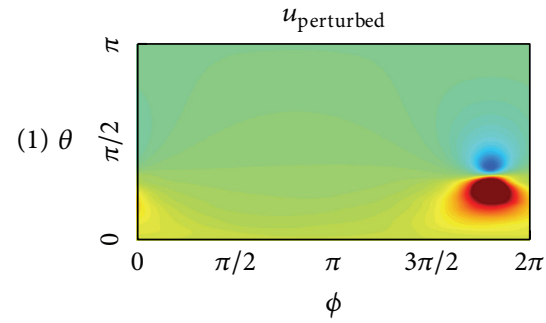

(a)

(2) $\theta$

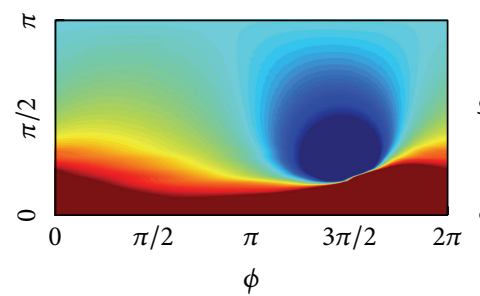

(d)

(3) $\theta$

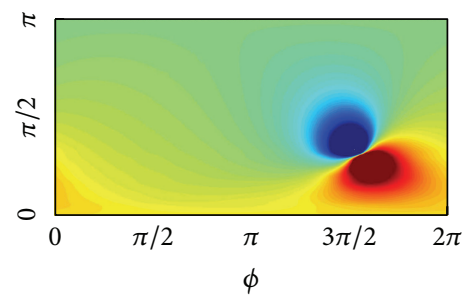

(g)

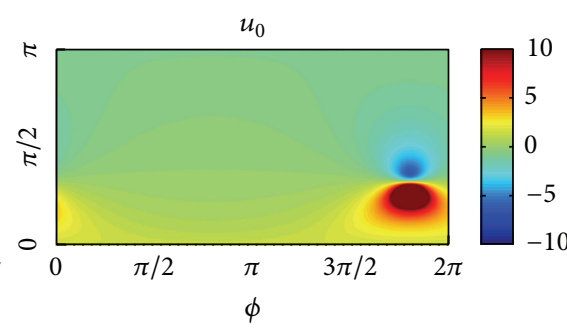

(b)

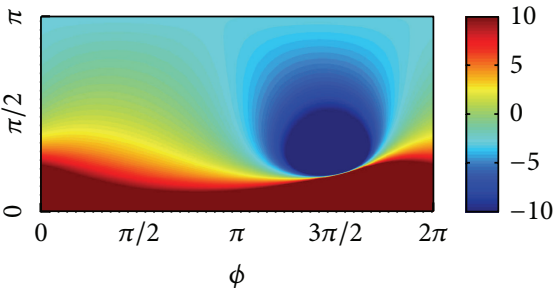

(e)

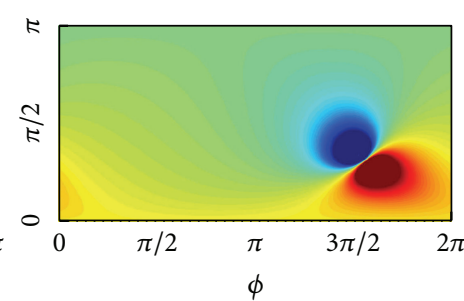

(h)

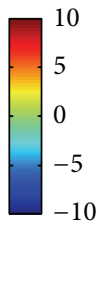

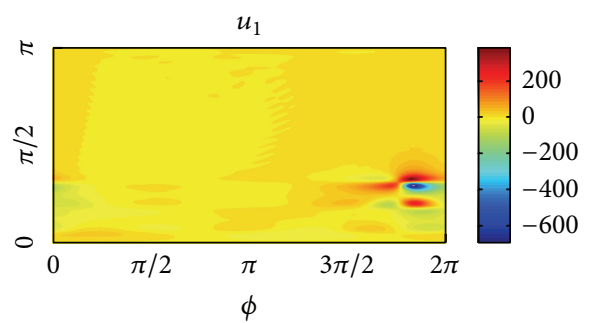

(c)

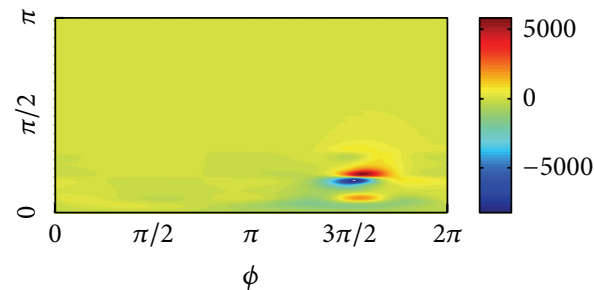

(f)

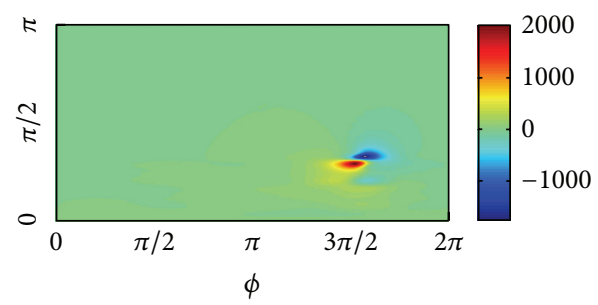

(i)

FIGURE 6: The electric surface potential ( $\mathrm{mV}$ s) originating from a spherical homogeneous conductor including a perturbed upper part (left column) as described in Section 4.2. The firing of neurons take place in the northern hemisphere, namely, at $(1) r_{0}=0.06 \mathrm{~m}, \theta_{0}=0.29 \pi$, $\phi_{0}=0.6 \pi$, and $\mathbf{Q}=(0,0,1) \times 10^{-5} ;(2) r_{0}=0.06 \mathrm{~m}, \theta_{0}=0.17 \pi, \phi_{0}=1.55 \pi$, and $\mathbf{Q}=(2,3,5) \times 10^{-5}$; and $(3) r_{0}=0.06 \mathrm{~m}$ and $\theta_{0}=0.32 \pi$, $\phi_{0}=1.55 \pi$. The corresponding recording concerning the unperturbed instance $u_{0}$ is shown in the middle column for the same dipole configuration, whereas the right column displays the contribution of the first-order correction $u_{1}$ on $u_{\text {perturbed }}=u\left(\mathbf{r}^{p}\right)$ as expressed by (32) for $\epsilon=0.004$.

alterations of the surface inflicted by external forces such as a blow or bump to the head, related to falls, vehicle accidents, sports, and violence; (2) deformations of the form of the human head due to natural mechanisms, especially the upper part where EEG signals are recorded.

We then pursue a solution having the form

$$
u\left(\mathbf{r}^{p}\right)=u_{0}(a \widehat{\mathbf{r}})+\epsilon u_{1}(a \widehat{\mathbf{r}})+\mathcal{O}\left(\epsilon^{2}\right),
$$

where the leading term $u_{0}$ provides the electric surface potential to the unperturbed case. The first-order approximation $u_{1}$ is then computed recursively. Whereas the zeroth-order solution $u_{0}$ can be expressed in closed form, the resulting relation for $u_{1}$ consists of a sum over five indices involving, in addition, integrals implicating the triple product of spherical harmonics. In all cases, the evaluation of the first-order approximation returns higher values in contrast to $u_{0}$ which are usually one to three orders of magnitude lower (see Figure 8, e.g.). Moreover, Figure 8 visually demonstrates the layered structure of (32) where the electric potential on the surface of the perturbed head is build by consecutively adding layers of approximations.
The present investigation is built upon a homogeneous spherical conductor linked to a dipolar source model for the simple reason that it permits the derivation of analytical formulas allowing an in-deep examination of every introduced parameter on both forward and inverse Electro- and Magnetoencephalography problems. Regarding the forward problem, (19) supports the installation of specific deformations through $f(\theta, \phi)$. On the other hand, the perturbation parameter $\epsilon$ controls independently of the chosen surface the depth/height of the deformation. Moreover, an isolated inspection of the first-order approximation $u_{1}$ reveals whenever surface deformations will have an impact on EEG measurements. The extent of this impact depends on the perturbation parameter $\epsilon$. Hence, the product $\epsilon u_{1}$ serves as a criterion in order to validate the significance of head shape deformations on EEG recordings. With respect to the inverse EEG problem a thorough examination based on the formulas presented in this work reveals that variations in the shape of the head influences not only the position of the dipolar source but the moment as well [27]. Obviously, this model is of course an approximation in itself. 


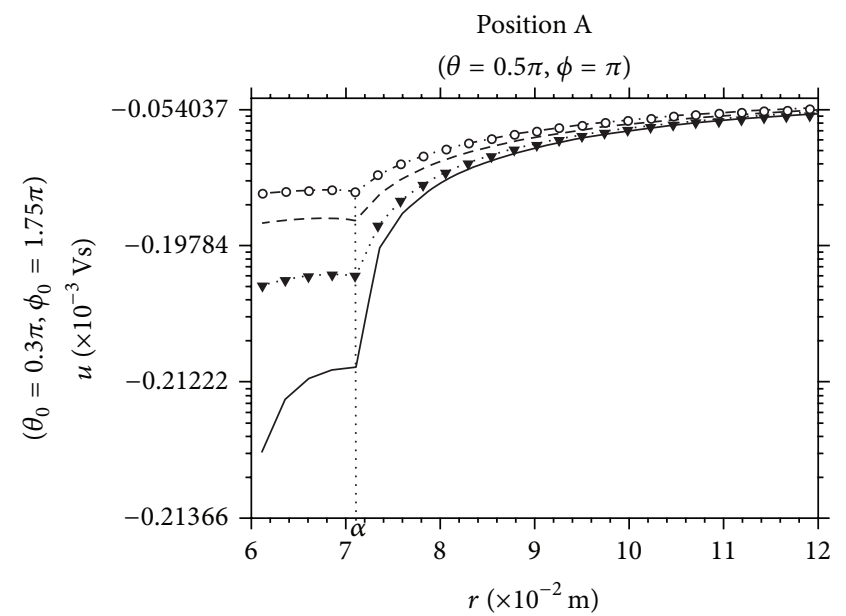

(a)

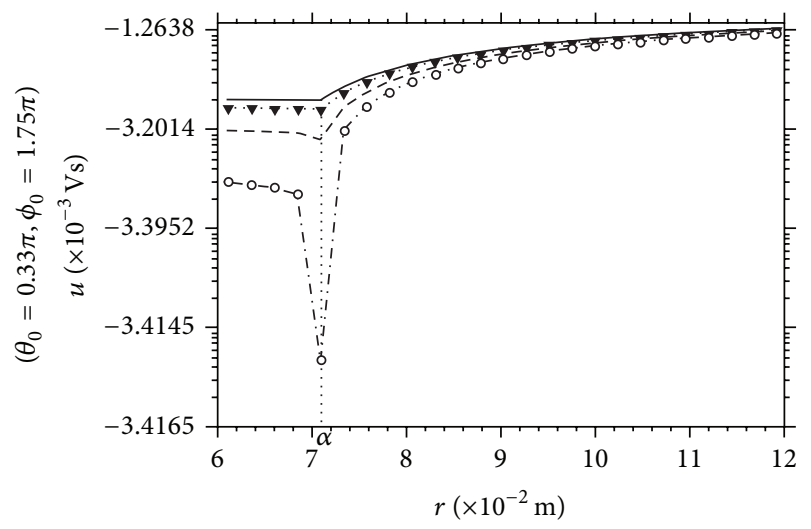

(c)

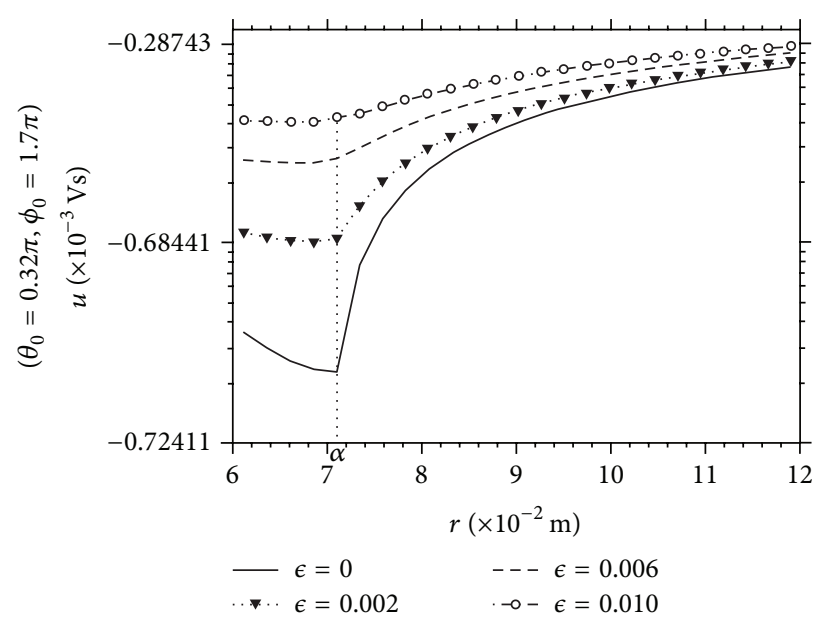

(e)

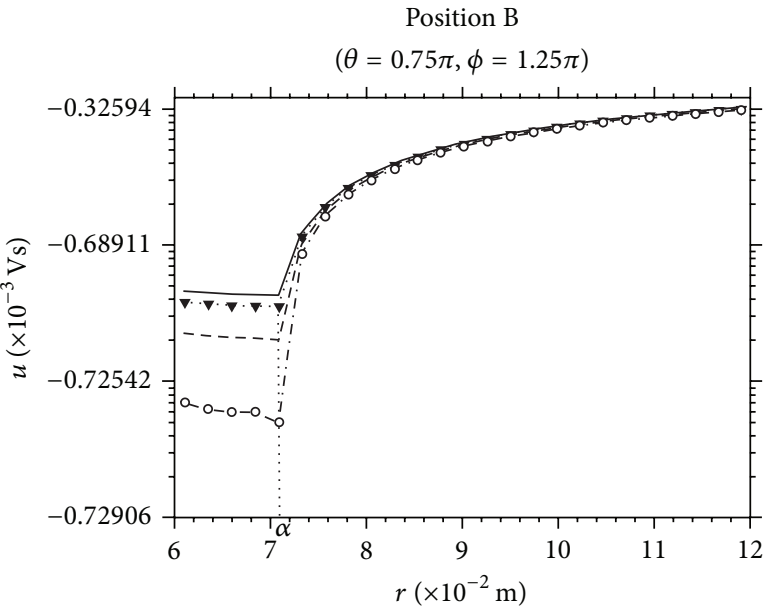

(b)

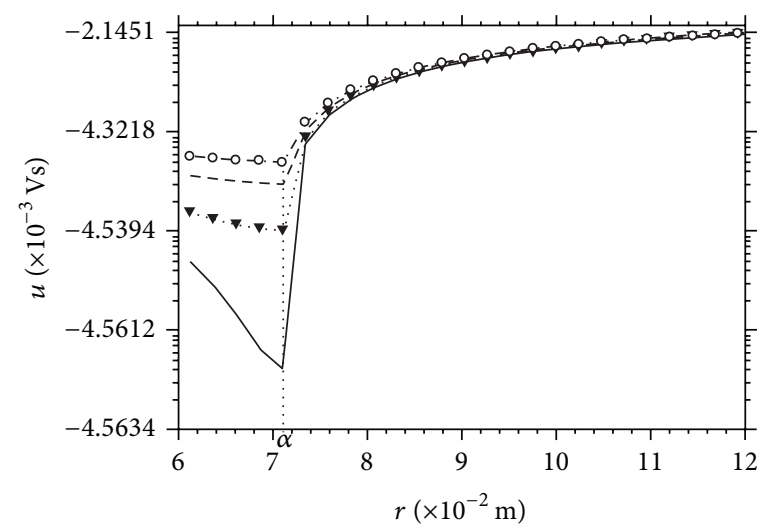

(d)

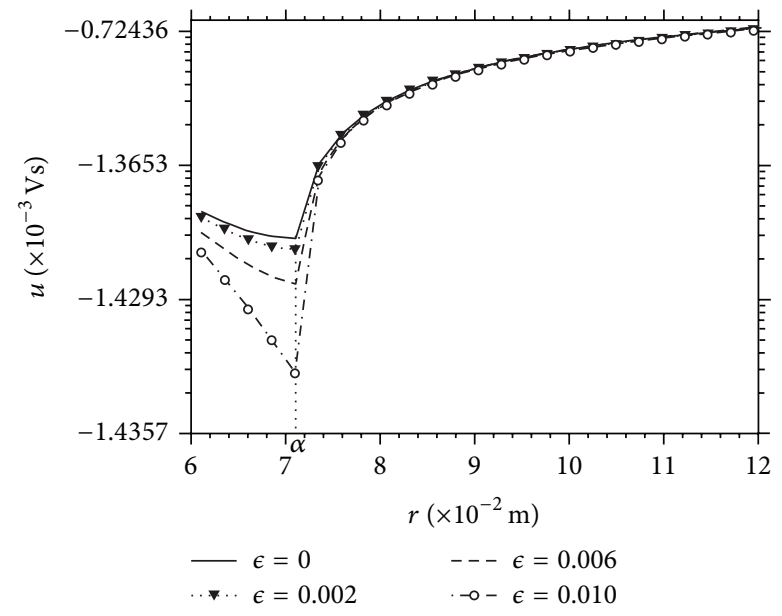

(f)

Figure 7: The electric potential on the surface $r^{p}(\theta, \phi ; \epsilon)$ and surroundings, along the rays $\theta=0.5 \pi, \phi=\pi$ (position A, left column) and $\theta=0.75 \pi, \phi=1.25 \pi$ (position B, right column), respectively, for distinct values of the perturbation parameter $\epsilon$, depending on the irregularities in appearance of the upper part of the human cranium as displayed in Figure 3. Again, each row represents a specific dipole arrangement, namely, (1) positioned at $r_{0}=0.06 \mathrm{~m}$ and $\theta_{0}=0.29 \pi, \phi_{0}=1.65 \pi$ with moments $\mathbf{Q}=(0,0,1) \times 10^{-5} \mathrm{Cm} ;(2)$ at $r_{0}=0.06 \mathrm{~m}$, $\theta_{0}=0.17 \pi, \phi_{0}=1.55 \pi$, and $\mathbf{Q}=(2,3,5) \times 10^{-5} \mathrm{Cm}$; (3) situated at $r_{0}=0.06 \mathrm{~m}, \theta_{0}=0.32 \pi, \phi_{0}=1.55 \pi$, and $\mathbf{Q}=(1,1,1) \times 10^{-5} \mathrm{Cm}$. 


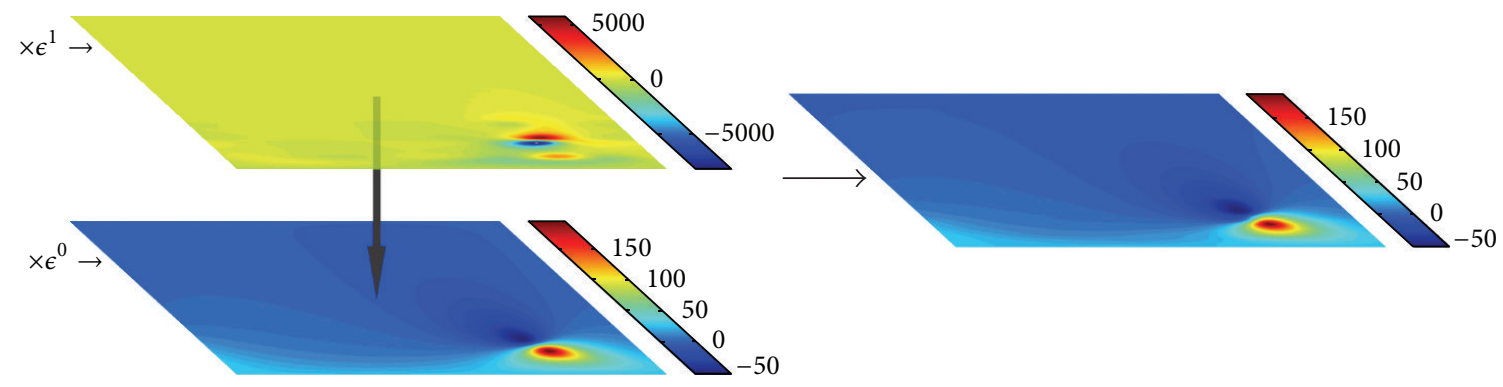

FIGURE 8: The electric potential on the surface of the perturbed head (right) constructed by adding to the corresponding potential for the unperturbed conductor (bottom left) the correction $\epsilon u_{1}$ (top left).

The results indicate that surface deformations alter the EEG measurements in some cases significantly if the active brain region is situated near the deformed area. We conclude that the accurate description of the head shape plays an important role in understanding the forward EEG problem in particular instances only. These situations can be identified with the aid of the above-mentioned criterion. On the other hand, since the behavior of the external potential is dictated by (14), that is, declines proportional to the inverse second power of the distance, the influence of geometric alterations, independent of shape, is negligible after four to five centimeters away from the surface (Figures 5 and 7). At this distance, the magnetic fields produced by neuronal activity of the brain (MEG) are registered and a simultaneous survey would be beneficial. However, considering that the Electroencephalographic readings a few centimeters above the scalp are near zero, a device capable of capturing these weak fields is needed.

\section{Appendices}

\section{A. Boundary Conditions on a Perturbed Sphere}

In order to identify the implication of the deformed boundary on the solution process, expansion (21) is assumed to be valid all the way to the boundary. The Neumann condition (11) now reads

$$
\widehat{\mathbf{n}} \cdot \nabla v\left(\mathbf{r}^{p}\right)=0, \quad \mathbf{r}^{p} \in \partial D^{p}
$$

where $\widehat{\mathbf{n}}$ is the unit outward normal to the perturbed boundary.

After simple manipulations relation (A.1) simplifies as

$$
\begin{aligned}
\left(\mathbf{r}^{p}\right)^{2} & \frac{\partial v\left(\mathbf{r}^{p}\right)}{\partial r}-\frac{\partial r^{p}}{\partial \theta} \frac{\partial v\left(\mathbf{r}^{p}\right)}{\partial \theta} \\
& -\frac{1}{\sin ^{2} \theta} \frac{\partial r^{p}}{\partial \phi} \frac{\partial v\left(\mathbf{r}^{p}\right)}{\partial \phi}=0, \quad \mathbf{r}^{p} \in \partial D^{p} .
\end{aligned}
$$

The above relation, combined with expressions (19) and (20), along with equating coefficients of $\epsilon$ and bearing in mind that $\partial_{r} f=0$, gives rise to the system

$$
\begin{aligned}
\widehat{\mathbf{r}} \cdot \nabla v_{n}^{-} & \left(a \widehat{\mathbf{r}}, \mathbf{r}_{0}\right) \\
& =\sum_{k=0}^{n-1}(-1)^{k}(k+1)\left(\frac{f}{a}\right)^{k}\left(\nabla f \cdot \nabla v_{n-k-1}^{-}\left(a \widehat{\mathbf{r}}, \mathbf{r}_{0}\right)\right),
\end{aligned}
$$

the latter holding for every $n$.

On the other hand, the boundary condition regarding the exterior electric potential (13) transforms as

$$
v_{n}^{+}\left(a \widehat{\mathbf{r}}, \mathbf{r}_{0}\right)=v_{n}^{-}\left(a \widehat{\mathbf{r}}, \mathbf{r}_{0}\right), \quad n=0,1,2, \ldots,
$$

accompanied by an expression similar to (14) for every $n$.

\section{B. Computing the First-Order Correction}

Customarily, a solution is pursued in form of a expansion in spherical harmonics, namely,

$$
v_{1}^{-}\left(\mathbf{r}, \mathbf{r}_{0}\right)=\sum_{n=0}^{\infty} \sum_{m=-n}^{n} \mathscr{B}_{n}^{m} r^{n} Y_{n}^{m}(\widehat{\mathbf{r}}) \text {. }
$$

The boundary condition (25) with the aid of (B.1) reads

$$
\begin{aligned}
\sum_{n=1}^{\infty} \sum_{m=-n}^{n} n a^{n+1} \mathscr{B}_{n}^{m} Y_{n}^{m}(\widehat{\mathbf{r}}) \\
=\frac{\partial f}{\partial \theta} \sum_{n=1}^{\infty} \sum_{m=-n}^{n} \frac{r_{0}^{n} \bar{Y}_{n}^{m}\left(\widehat{\mathbf{r}}_{0}\right)}{n a^{n+1}}\left(\frac{\partial}{\partial \theta} Y_{n}^{m}(\widehat{\mathbf{r}})\right) \\
+\frac{1}{\sin ^{2} \theta} \frac{\partial f}{\partial \phi} \sum_{n=1}^{\infty} \sum_{m=-n}^{n} \frac{r_{0}^{n} \bar{Y}_{n}^{m}\left(\widehat{\mathbf{r}}_{0}\right)}{n a^{n+1}}\left(\frac{\partial}{\partial \phi} Y_{n}^{m}(\widehat{\mathbf{r}})\right) .
\end{aligned}
$$

Given that

$$
\begin{aligned}
\sin \theta & \frac{\partial}{\partial \theta} Y_{n}^{m}(\widehat{\mathbf{r}}) \\
& =n j_{n+1}^{m} Y_{n+1}^{m}(\widehat{\mathbf{r}})-(n+1) j_{n}^{m} Y_{n-1}^{m}(\widehat{\mathbf{r}}),
\end{aligned}
$$


where

$$
\begin{gathered}
j_{n}^{m}=\sqrt{\frac{n^{2}-m^{2}}{4 n^{2}-1}}, \\
\frac{\partial}{\partial \phi} Y_{n}^{m}(\widehat{\mathbf{r}})=i m Y_{n}^{m}(\widehat{\mathbf{r}}),
\end{gathered}
$$

relation (B.2) becomes

$$
\begin{aligned}
\sum_{n=1}^{\infty} \sum_{m=-n}^{n} n a^{n+1} \mathscr{B}_{n}^{m} Y_{n}^{m}(\widehat{\mathbf{r}}) \\
=\frac{1}{\sin \theta} \frac{\partial f}{\partial \theta} \\
\quad \times \sum_{n=1}^{\infty} \sum_{m=-n}^{n} \frac{r_{0}^{n} \bar{Y}_{n}^{m}\left(\widehat{\mathbf{r}}_{0}\right)}{n a^{n+1}} \\
\quad+\frac{i}{\sin ^{2} \theta} \frac{\partial f}{\partial \phi} \sum_{n=1}^{\infty} \sum_{m=-n}^{n} \frac{m}{n} \frac{r_{0}^{n} \bar{Y}_{n}^{m}\left(\widehat{\mathbf{r}}_{0}\right)}{a^{n+1}} Y_{n}^{m}(\widehat{\mathbf{r}}) .
\end{aligned}
$$

Rather expanding the function $f(\theta, \phi)$ in terms of spherical harmonics it is preferable to expand the products

$$
\frac{1}{\sin \theta} \frac{\partial f}{\partial \theta}, \quad \frac{1}{\sin ^{2} \theta} \frac{\partial f}{\partial \phi}
$$

instead, and relation (B.5) is rewritten as

$$
\begin{aligned}
& \sum_{n=1}^{\infty} \sum_{m=-n}^{n} n a^{n+1} \mathscr{B}_{n}^{m} Y_{n}^{m}(\widehat{\mathbf{r}}) \\
& =\frac{1}{\sqrt{4 \pi}} \sum_{n=1}^{\infty} \sum_{m=-n}^{n} \frac{r_{0}^{n} \bar{Y}_{n}^{m}\left(\widehat{\mathbf{r}}_{0}\right)}{n a^{n+1}} \\
& \cdot\left[\beta _ { 0 } ^ { 0 } \left(n j_{n+1}^{m} Y_{n+1}^{m}(\widehat{\mathbf{r}})\right.\right. \\
& \left.\quad-(n+1) j_{n}^{m} Y_{n-1}^{m}(\widehat{\mathbf{r}})\right) \\
& +\sum_{n=1}^{\infty} \sum_{k=1}^{n} \sum_{m=-k}^{k} \sum_{\ell=-s}^{s} \frac{r_{0}^{k} \bar{Y}_{k}^{m}\left(\widehat{\mathbf{r}}_{0}\right)}{a^{k+1} j_{k+1}^{m} \beta_{s}^{\ell} Y_{k+1}^{m}(\widehat{\mathbf{r}}) Y_{s}^{\ell}(\widehat{\mathbf{r}})} \\
& +\sum_{n=1}^{\infty} \sum_{k=1}^{n} \sum_{m=-k}^{k} \sum_{\ell=-s}^{s} \frac{\left.r_{0}^{k} \bar{Y}_{k}^{m}(\widehat{\mathbf{r}})\right]}{k a^{k+1}} \\
& \cdot\left[\begin{array}{l}
-(k+1) j_{k}^{m} \beta_{s}^{\ell} Y_{k-1}^{m}(\widehat{\mathbf{r}}) \\
\left.\times Y_{s}^{\ell}(\widehat{\mathbf{r}})+i m \gamma_{s}^{\ell} Y_{k}^{m}(\widehat{\mathbf{r}}) Y_{s}^{\ell}(\widehat{\mathbf{r}})\right]
\end{array}\right.
\end{aligned}
$$

where $s=n-k+1$ and

$$
\begin{aligned}
\beta_{s}^{\ell} & =\oint_{S^{2}} \frac{1}{\sin \theta} \frac{\partial f(\widehat{\mathbf{r}})}{\partial \theta} \bar{Y}_{s}^{\ell}(\widehat{\mathbf{r}}) \mathrm{d} \Omega(\widehat{\mathbf{r}}), \\
\gamma_{s}^{\ell} & =\oint_{S^{2}} \frac{1}{\sin ^{2} \theta} \frac{\partial f(\widehat{\mathbf{r}})}{\partial \phi} \bar{Y}_{s}^{\ell}(\widehat{\mathbf{r}}) \mathrm{d} \Omega(\widehat{\mathbf{r}}),
\end{aligned}
$$

where $\mathrm{d} \Omega(\widehat{\mathbf{r}})=\sin \theta \mathrm{d} \theta \mathrm{d} \phi$ denotes the solid angle element.

Multiplying the latter throughout $\bar{Y}_{p}^{q}(\widehat{\mathbf{r}})$ and integrating over the unit ball yields

$$
\begin{aligned}
& \mathscr{B}_{p}^{q} p a^{p+1} \\
& =\mathscr{D}_{p}^{q}+\sum_{n=1}^{\infty} \sum_{k=1}^{n} \sum_{m=-k}^{k} \sum_{\ell=-s}^{s} \frac{r_{0}^{k} \bar{Y}_{k}^{m}\left(\widehat{\mathbf{r}}_{0}\right)}{a^{k+1}} j_{k+1}^{m} \beta_{s}^{\ell} \\
& \times \oint_{S^{2}} Y_{k+1}^{m}(\widehat{\mathbf{r}}) Y_{s}^{\ell}(\widehat{\mathbf{r}}) \bar{Y}_{p}^{q}(\widehat{\mathbf{r}}) \mathrm{d} \Omega(\widehat{\mathbf{r}}) \\
& +\sum_{n=1}^{\infty} \sum_{k=1}^{n} \sum_{m=-k} \sum_{\ell=-s}^{s} \frac{r_{0}^{k} \bar{Y}_{k}^{m}\left(\widehat{\mathbf{r}}_{0}\right)}{k a^{k+1}} \\
& \times\left[-(k+1) j_{k}^{m} \beta_{s}^{\ell}\right. \\
& \quad \oint_{S^{2}} Y_{k-1}^{m}(\widehat{\mathbf{r}}) Y_{s}^{\ell}(\widehat{\mathbf{r}}) \bar{Y}_{p}^{q}(\widehat{\mathbf{r}}) \mathrm{d} \Omega(\widehat{\mathbf{r}}) \\
& \left.+i m \gamma_{s}^{\ell} \oint_{S^{2}} Y_{k}^{m}(\widehat{\mathbf{r}}) Y_{s}^{\ell}(\widehat{\mathbf{r}}) \bar{Y}_{p}^{q}(\widehat{\mathbf{r}}) \mathrm{d} \Omega(\widehat{\mathbf{r}})\right]
\end{aligned}
$$

in as much as

$$
\oint_{S^{2}} Y_{n}^{m}(\widehat{\mathbf{r}}) \bar{Y}_{p}^{q}(\widehat{\mathbf{r}}) \mathrm{d} \Omega(\widehat{\mathbf{r}})=\delta_{n, p} \delta_{m, q}
$$

where $\delta_{i, j}$ is the Kronecker delta, and the coefficients $\mathscr{D}_{p}^{q}$ are expressed via (29).

Integration concerning the triple product of spherical harmonics over the unit ball is connected with the notion of the so-called Wigner-3j symbol [28, Chapter 34]

$$
\left(\begin{array}{ccc}
n_{1} & n_{2} & n_{3} \\
m_{1} & m_{2} & m_{3}
\end{array}\right)
$$

provided that, first and foremost, $n_{1}, n_{2}, n_{3}$ are positive integers, $m_{1}+m_{2}+m_{3}=0$ in connection to the triangle condition $\left|n_{r}-n_{s}\right| \leq n_{t} \leq n_{r}+n_{s}$, where $r, s, t$ is any permutation of $1,2,3$.

Consequently

$$
\begin{aligned}
\oint_{S^{2}} Y_{n_{1}}^{m_{1}}(\widehat{\mathbf{r}}) Y_{n_{2}}^{m_{2}}(\widehat{\mathbf{r}}) Y_{n_{3}}^{m_{3}}(\widehat{\mathbf{r}}) \mathrm{d} \Omega(\widehat{\mathbf{r}}) \\
=K_{n_{1}, n_{2}, n_{3}} \\
\quad \times\left(\begin{array}{ccc}
n_{1} & n_{2} & n_{3} \\
0 & 0 & 0
\end{array}\right)\left(\begin{array}{ccc}
n_{1} & n_{2} & n_{3} \\
m_{1} & m_{2} & m_{3}
\end{array}\right),
\end{aligned}
$$

where

$$
K_{n_{1}, n_{2}, n_{3}}=\sqrt{\frac{\left(2 n_{1}+1\right)\left(2 n_{2}+1\right)\left(2 n_{3}+1\right)}{4 \pi}} .
$$


On that account and bearing in mind that

$$
\bar{Y}_{p}^{q}(\widehat{\mathbf{r}})=(-1)^{q} Y_{p}^{-q}(\widehat{\mathbf{r}})
$$

we find

$$
\begin{aligned}
\oint_{S^{2}} Y_{e}^{m}(\widehat{\mathbf{r}}) Y_{s}^{\ell}(\widehat{\mathbf{r}}) Y_{p}^{-q}(\widehat{\mathbf{r}}) \mathrm{d} \Omega(\widehat{\mathbf{r}}) \\
=(-1)^{q} K_{e, s, p} \\
\quad \times\left(\begin{array}{ccc}
e & s & p \\
0 & 0 & 0
\end{array}\right)\left(\begin{array}{ccc}
e & s & p \\
m & \ell & -q
\end{array}\right),
\end{aligned}
$$

where $e$ is either $k-1, k$ or $k+1$.

Replacing everything into (B.10) we get

$$
\begin{aligned}
& \mathscr{B}_{p}^{q}=\frac{1}{p a^{p+1}} \mathscr{D}_{p}^{q}+\frac{(-1)^{q}}{p a^{p+1}} \\
& \times\left[\sum_{n=1}^{\infty} \sum_{k=1}^{n} \sum_{m=-k}^{k} \frac{r_{0}^{k} \bar{Y}_{k}^{m}\left(\widehat{\mathbf{r}}_{0}\right)}{a^{k+1}} \beta_{s}^{q-m} j_{k+1}^{m} K_{k+1, s, p}\right. \\
& \times\left(\begin{array}{ccc}
k+1 & s & p \\
0 & 0 & 0
\end{array}\right)\left(\begin{array}{ccc}
k+1 & s & p \\
m & q-m & -q
\end{array}\right) \\
& -\sum_{n=1}^{\infty} \sum_{k=1}^{n} \sum_{m=-k}^{k} \frac{k+1}{k} \frac{r_{0}^{k} \bar{Y}_{k}^{m}\left(\widehat{\mathbf{r}}_{0}\right)}{a^{k+1}} \beta_{s}^{q-m} j_{k}^{m} K_{k-1, s, p} \\
& \times\left(\begin{array}{ccc}
k-1 & s & p \\
0 & 0 & 0
\end{array}\right)\left(\begin{array}{ccc}
k-1 & s & p \\
m & q-m & -q
\end{array}\right) \\
& +i \sum_{n=1}^{\infty} \sum_{k=1}^{n} \sum_{m=-k}^{k} \frac{m}{k} \frac{r_{0}^{k} \bar{Y}_{k}^{m}\left(\widehat{\mathbf{r}}_{0}\right)}{a^{k+1}} \gamma_{s}^{q-m} K_{k, s, p} \\
& \left.\times\left(\begin{array}{ccc}
k & s & p \\
0 & 0 & 0
\end{array}\right)\left(\begin{array}{ccc}
k & s & p \\
m & q-m & -q
\end{array}\right)\right] \quad p \geq 1,
\end{aligned}
$$

where we took advantage of the fact that $m+\ell-q$ vanishes canceling the fourth of the expansions. In addition, the triangular condition

$$
|(k+1)-(n-k+1)| \leq p \leq(k+1)+(n-k+1)
$$

for the Wigner-3j symbols present in (B.17) as well as

$$
\begin{gathered}
|(k-1)-(n-k+1)| \leq p \leq(k-1)+(n-k+1) \\
|k-(n-k+1)| \leq p \leq k+(n-k+1)
\end{gathered}
$$

for the remaining two provides a lower bound for the index $n$, namely, $n \geq p-2, n \geq p$, and $n \geq p-1$ for the first, second, and third expansion, respectively.

Putting everything together, relation (B.1) reads

$$
v_{1}\left(\mathbf{r}, \mathbf{r}_{0}\right)=\sum_{p=1}^{\infty} \sum_{q=-p}^{p} \mathscr{B}_{p}^{q}\left(\mathbf{r}_{0}\right) r^{p} Y_{p}^{q}(\widehat{\mathbf{r}}) .
$$

Employing the fact that the first of Wigner-3j symbols present in (B.13) vanish if $n_{1}+n_{2}+n_{3}$ is odd, it can be shown that

$$
\mathscr{B}_{p}^{-q}=(-1)^{q} \overline{\mathscr{B}}_{p}^{q}
$$

leading to (27).

Since $v_{1}$ depends on the indices $p$ and $n$ an important question refers to finding suitable values for those parameters in order for $v_{1}$ to converge. A slow convergences is expected since the source is situated close to the surface. Numerical simulations reveal that convergence is achieved for values of $p$ higher than 50 and $n$ over 60 .

\section{Conflict of Interests}

The authors declare that there is no conflict of interests regarding the publication of this paper.

\section{Acknowledgments}

The authors gratefully acknowledge the contribution of the "ARISTEIA" Action of the "OPERATIONAL PROGRAMME EDUCATION AND LIFELONG LEARNING” cofunded by the European Social Fund (ESF) and National Resources. The authors would also like to thank Kamvyssas Gregory and Hadjinicolaou Maria for participating in discussions.

\section{References}

[1] F. Kariotou, "On the mathematics of EEG and MEG in spheroidal geometry," Bulletin of the Greek Mathematical Society, vol. 47, pp. 117-135, 2003.

[2] F. Kariotou, "Electroencephalography in ellipsoidal geometry," Journal of Mathematical Analysis and Applications, vol. 290, no. 1, pp. 324-342, 2004.

[3] D. Yao, "Electric potential produced by a dipole in a homogeneous conducting sphere," IEEE Transactions on Biomedical Engineering, vol. 47, no. 7, pp. 964-966, 2000.

[4] G. Dassios, "Electric and magnetic activity of the brain in spherical and ellipsoidal geometry," in Mathematical Modeling in Biomedical Imaging I, H. Ammari, Ed., Lecture Notes in Mathematics, pp. 133-202, Springer, Berlin, Germany, 1983.

[5] H. G. Wieser, K. Schindler, and D. Zumsteg, "EEG in Creutzfeldt-Jakob disease," Clinical Neurophysiology, vol. 117, no. 5, pp. 935-951, 2006.

[6] U. R. Acharya, S. V. Sree, G. Swapna, R. J. Martis, and J. S. Suri, "Automated EEG analysis of epilepsy: a review," KnowledgeBased Systems, vol. 45, pp. 147-165, 2013.

[7] B. N. Cuffin, "Effects of head shape on EEGs and MEGs," IEEE Transactions on Biomedical Engineering, vol. 37, no. 1, pp. 44-52, 1990.

[8] B. N. Cuffin, "Effects of local variations in skull and scalp thickness on EEG's and MEG's," IEEE Transactions on Biomedical Engineering, vol. 40, no. 1, pp. 42-48, 1993.

[9] N. Chauveau, X. Franceries, B. Doyon, B. Rigaud, J. P. Morucci, and P. Celsis, "Effects of skull thickness, anisotropy, and inhomogeneity on forward EEG/ERP computations using a spherical three-dimensional resistor mesh model," Human Brain Mapping, vol. 21, no. 2, pp. 86-97, 2004. 
[10] F. Zanow and M. J. Peters, "Individually shaped volume conductor models of the head in EEG source localisation," Medical and Biological Engineering and Computing, vol. 33, no. 4, pp. 582588, 1995.

[11] N. von Ellenrieder, C. H. Muravchik, and A. Nehorai, "Effects of geometric head model perturbations on the EEG forward and inverse problems," IEEE Transactions on Biomedical Engineering, vol. 53, no. 3, pp. 421-429, 2006.

[12] N. Von Ellenrieder, C. H. Muravchik, M. Wagner, and A. Nehorai, "Effect of head shape variations among individuals on the EEG/MEG forward and inverse problems," IEEE Transactions on Biomedical Engineering, vol. 56, no. 3, pp. 587-597, 2009.

[13] B. Lanfer, M. Scherg, M. Dannhauer, T. R. Knösche, M. Burger, and C. H. Wolters, "Influences of skull segmentation inaccuracies on EEG source analysis," NeuroImage, vol. 62, no. 1, pp. 418-431, 2012.

[14] F. N. Wilson and R. H. Bayley, "The electric field of an eccentric dipole in a homogeneous spherical conducting medium," Circulation, vol. 1, no. 1, pp. 84-92, 1950.

[15] N. G. de Bruijn, Asymptotic Methods in Analysis, vol. 4 of Bibliotheca Mathematica, North-Holland, Amsterdam, The Netherlands, 1958.

[16] R. Bellman, Perturbation Techniques in Mathematics, Engineering and Physics, Holt Rinehart \& Winston, New York, NY, USA, 1966.

[17] G. Nolte and G. Curio, "Perturbative analytical solutions of the electric forward problem for realistic volume conductors," Journal of Applied Physics, vol. 86, no. 5, pp. 2800-2811, 1999.

[18] D. B. Geselowitz, "On bioelectric potentials in an inhomogeneous volume conductor," Biophysical Journal, vol. 7, no. 1, pp. $1-11,1967$.

[19] G. D. Murray, G. M. Teasdale, R. Braakman et al., "The European Brain Injury Consortium survey of head injuries," Acta Neurochirurgica, vol. 141, no. 3, pp. 223-236, 1999.

[20] L. G. Farkas, Anthropometry of the Head and Face, Raven Press, New York, NY, USA, 1994.

[21] R. Ball, C. Shu, P. Xi, M. Rioux, Y. Luximon, and J. Molenbroek, "A comparison between Chinese and Caucasian head shapes," Applied Ergonomics, vol. 41, no. 6, pp. 832-839, 2010.

[22] R. Plonsey and D. B. Heppner, "Considerations of quasistationarity in electrophysiological systems," The Bulletin of Mathematical Biophysics, vol. 29, no. 4, pp. 657-664, 1967.

[23] J. A. Stratton, Electromagnetic Theory, McGraw-Hill, New York, NY, USA, 1941.

[24] O. Gilad, L. Horesh, and D. S. Holder, "Design of electrodes and current limits for low frequency electrical impedance tomography of the brain," Medical and Biological Engineering \& Computing, vol. 45, no. 7, pp. 621-633, 2007.

[25] J.-F. P. J. Abascal, S. R. Arridge, D. Atkinson et al., "Use of anisotropic modelling in electrical impedance tomography; description of method and preliminary assessment of utility in imaging brain function in the adult human head," NeuroImage, vol. 43 , no. 2, pp. 258-268, 2008.

[26] H. Hallez, B. Vanrumste, R. Grech et al., "Review on solving the forward problem in EEG source analysis," Journal of NeuroEngineering and Rehabilitation, vol. 4, article 46, 2007.

[27] M. Doschoris and G. Dassios, "The influence of surface deformations on the source position and momentfor EEG," in preparation.

[28] F. W. J. Olver, D. W. Lozier, R. F. Boisvert, and C. W. Clark, Eds., NIST Handbook of Mathematical Functions, Cambridge University Press, Cambridge, UK, 2010. 


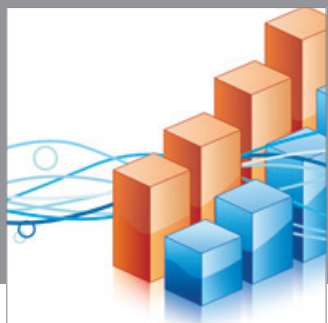

Advances in

Operations Research

mansans

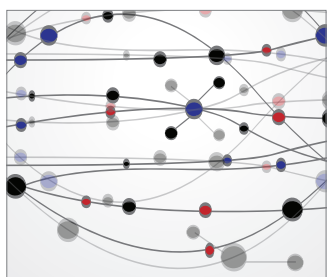

The Scientific World Journal
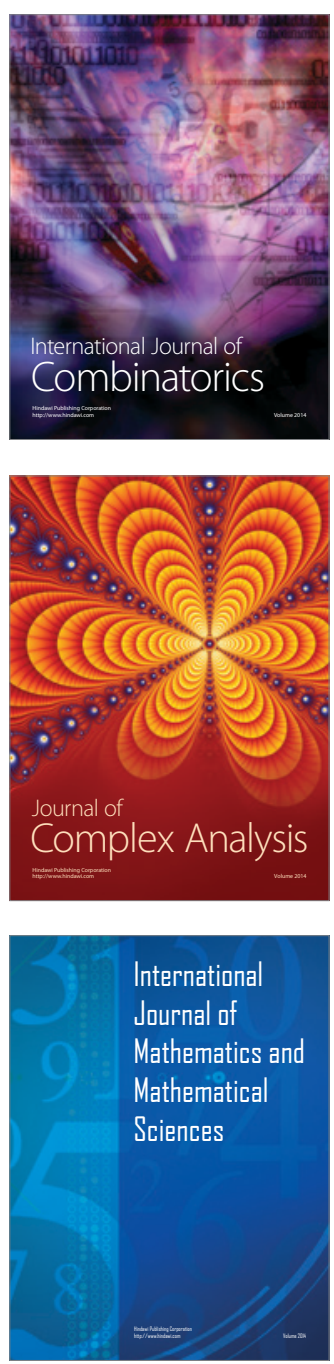
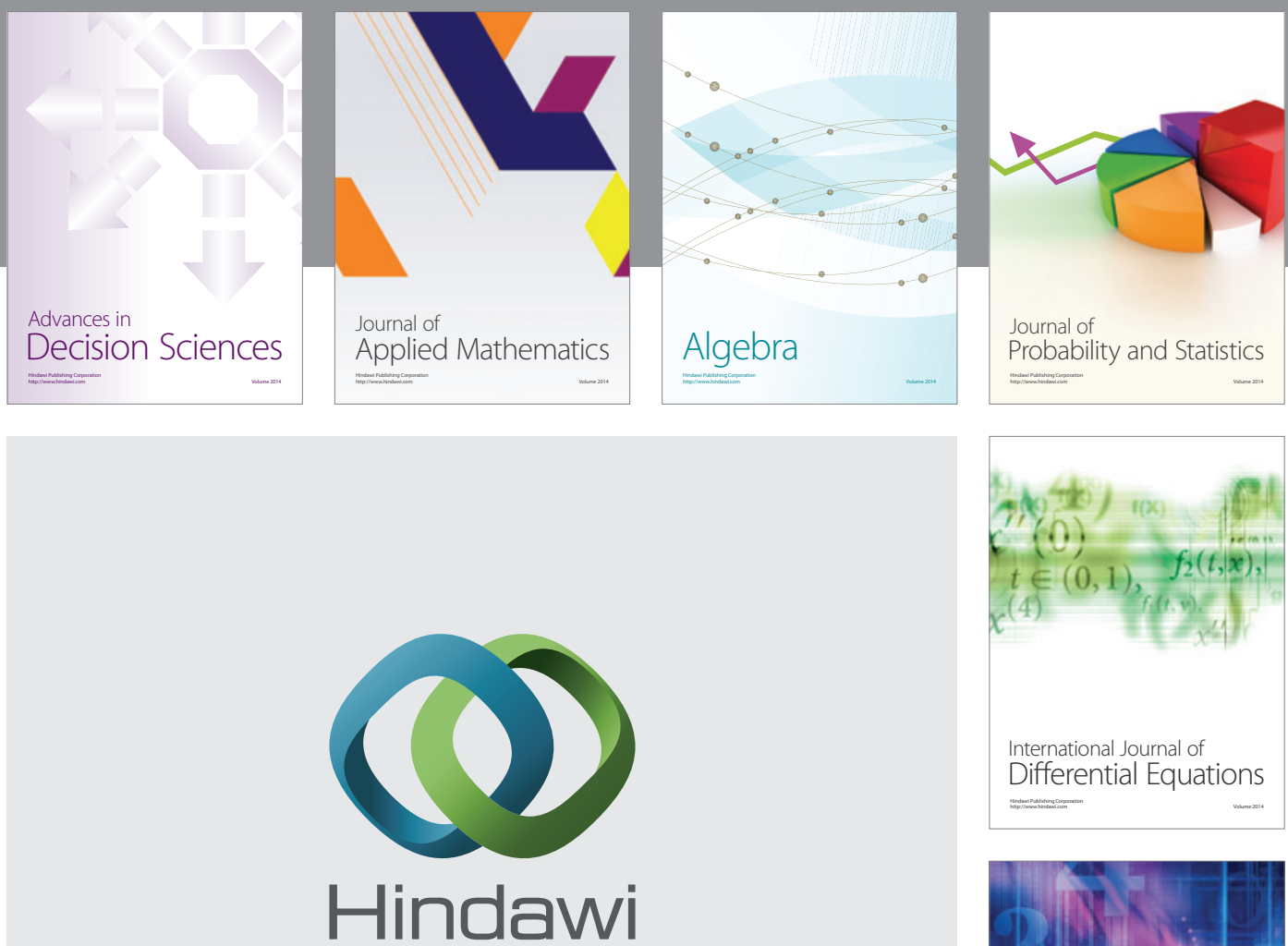

Submit your manuscripts at http://www.hindawi.com
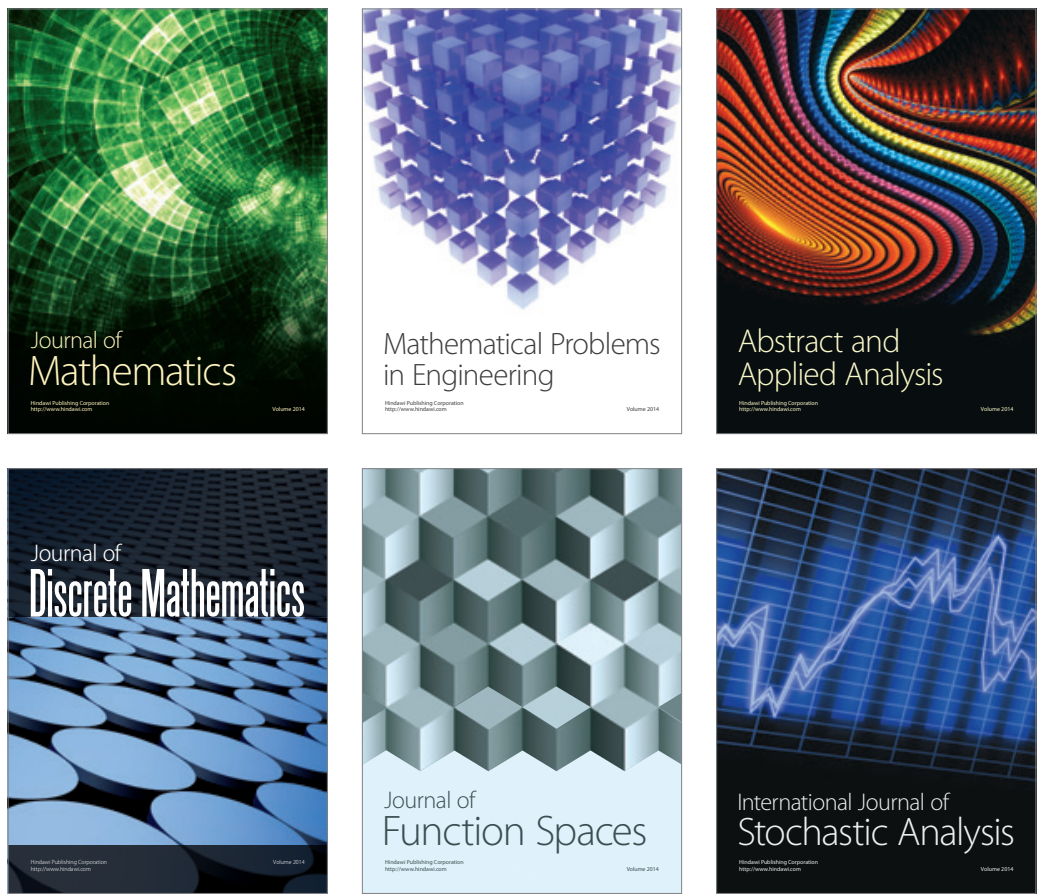

Journal of

Function Spaces

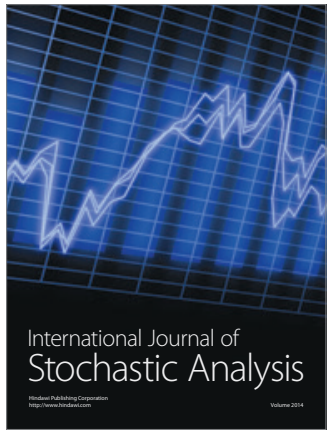

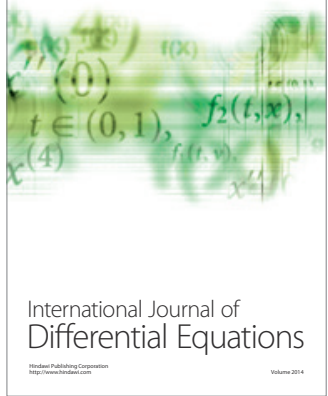
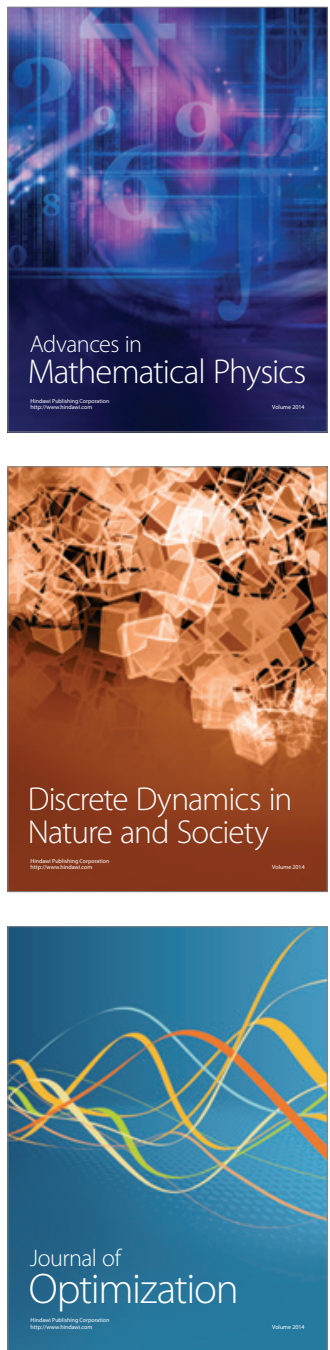